Supplement of Atmos. Chem. Phys., 21, 8323-8339, 2021

https://doi.org/10.5194/acp-21-8323-2021-supplement

(C) Author(s) 2021. CC BY 4.0 License.

(c) (1)
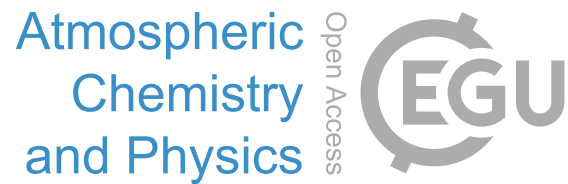

Supplement of

\title{
Increase in secondary organic aerosol in an urban environment
}

Marta Via et al.

Correspondence to: Marta Via (marta.via@idaea.csic.es) and María Cruz Minguillón (mariacruz.minguillon@idaea.csic.es)

The copyright of individual parts of the supplement might differ from the article licence. 


\section{Supplementary material}

Section 1. Supplementary tables and figures

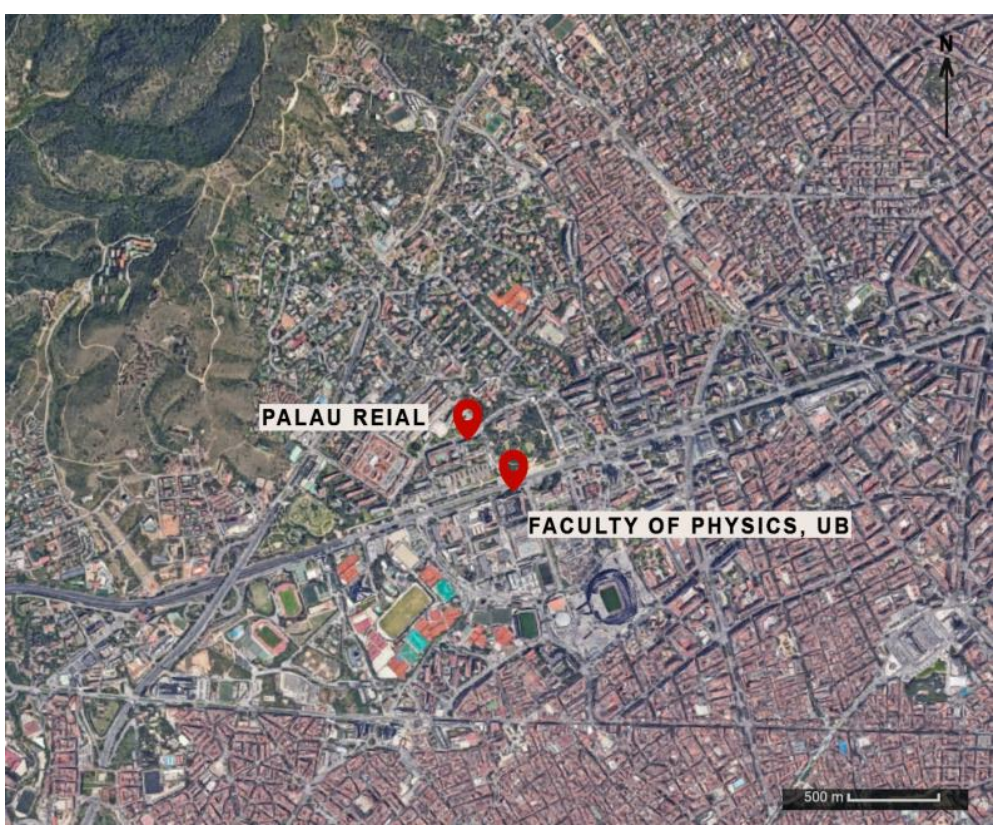

Map data @2021 Inst. Geogr. Nacional, Google, Institut Cartogràfic de Catalunya

5 Figure S 1. Location of Palau Reial and the Faculty of Physics in aerial view zoom to the site (Google Maps).
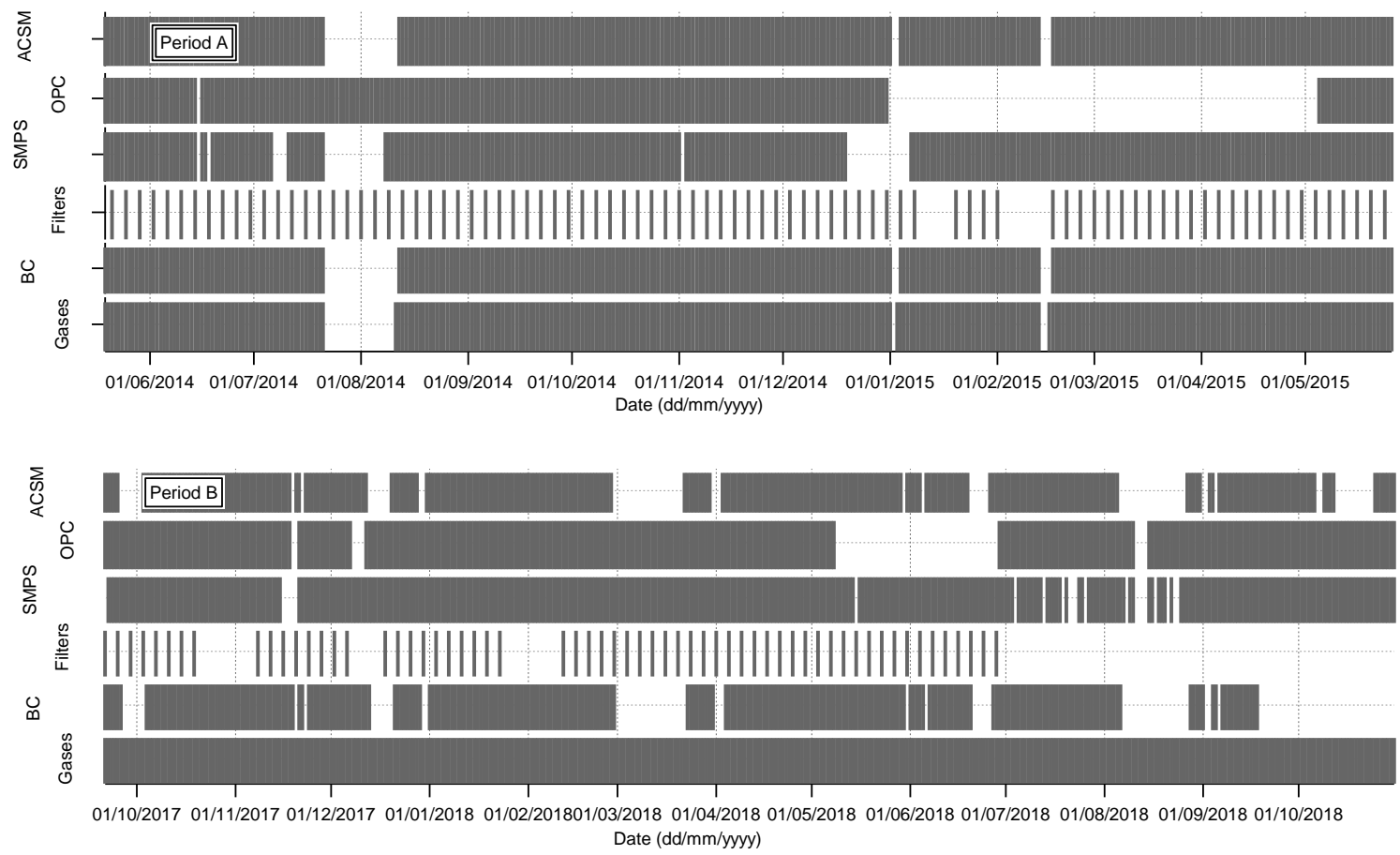

Figure S 2. Data availability for periods A and B. 
Table S 1. Output correlations of runs of different number of factors (n) per each season in periods A and B.

Bold rows correspond to those selected output runs.

\begin{tabular}{|c|c|c|c|c|c|c|c|c|c|c|c|c|}
\hline \multirow[b]{2}{*}{ A } & \multirow{2}{*}{ n } & \multirow{2}{*}{ Q/Qexp } & \multirow{2}{*}{$\begin{array}{c}\text { a-value } \\
\text { COA }\end{array}$} & \multirow{2}{*}{$\begin{array}{c}\text { a-value } \\
\text { HOA }\end{array}$} & \multirow{2}{*}{$\begin{array}{l}\text { a-value } \\
\text { BBOA }\end{array}$} & \multirow{2}{*}{$\begin{array}{c}\text { COA vs. } \\
\mathrm{m} / \mathrm{z} 55\end{array}$} & \multirow{2}{*}{$\begin{array}{c}\text { HOA } \\
\text { vs. BC }\end{array}$} & \multirow{2}{*}{$\begin{array}{c}\text { BBOA } \\
\text { vs. } \\
\mathrm{m} / \mathrm{z} 60\end{array}$} & \multirow{2}{*}{$\begin{array}{c}\text { LO-OO } \\
\text { A vs. } \\
\mathrm{NO}_{3}{ }^{-}\end{array}$} & \multirow{2}{*}{$\begin{array}{c}\text { MO-OO } \\
\mathrm{A} \text { vs. } \\
\mathrm{SO}_{4}{ }^{2-}\end{array}$} & \multicolumn{2}{|c|}{ OA meas. vs OA model } \\
\hline & & & & & & & & & & & $\mathbf{R}^{2}$ & Slope \\
\hline \multirow{3}{*}{$\begin{array}{l}\text { May } \\
2014\end{array}$} & 3 & $0.789 \pm 0.009$ & 0.3 & 0.3 & - & 0.56 & 0.63 & - & 0.44 & 0.04 & 0.94 & $1.023 \pm 0.011$ \\
\hline & 4 & $0.706 \pm 0.008$ & 0.5 & 0.1 & - & 0.60 & 0.67 & - & 0.37 & 0.21 & 0.96 & $0.996 \pm 0.008$ \\
\hline & 5 & $0.678 \pm 0.011$ & 0.2 & 0.5 & 0.1 & 0.61 & 0.65 & 0.28 & 0.47 & 0.10 & 0.98 & $1.029 \pm 0.008$ \\
\hline \multirow{3}{*}{$\begin{array}{l}\text { Jun-Aug } \\
2014\end{array}$} & 3 & $1.858 \pm 0.017$ & 0.1 & 0.5 & - & 0.77 & 0.41 & - & 0.27 & 0.12 & 0.95 & $1.039 \pm 0.004$ \\
\hline & 4 & $1.712 \pm 0.004$ & 0.1 & 0.5 & - & 0.76 & 0.37 & - & 0.25 & 0.18 & 0.96 & $1.035 \pm 0.004$ \\
\hline & 5 & $1.572 \pm 0.017$ & 0.2 & 0.5 & - & 0.74 & 0.34 & - & 0.23 & 0.16 & 0.96 & $1.025 \pm 0.003$ \\
\hline \multirow{3}{*}{$\begin{array}{l}\text { Sep-Oct } \\
2014\end{array}$} & 3 & $3.188 \pm 0.017$ & 0.1 & 0.5 & - & 0.66 & 0.68 & - & 0.35 & 0.07 & 0.95 & $\begin{array}{c}0.9545 \pm 0.00 \\
41\end{array}$ \\
\hline & 4 & $2.929 \pm 0.008$ & 0.1 & 0.3 & - & 0.35 & 0.68 & - & 0.35 & 0.08 & 0.96 & $0.968 \pm 0.003$ \\
\hline & 5 & $2.76 \pm 0.03$ & 0.1 & 0.5 & 0.5 & 0.64 & 0.68 & 0.4 & 0.32 & 0.09 & 0.97 & $0.991 \pm 0.004$ \\
\hline \multirow{3}{*}{$\begin{array}{c}\text { Nov-Mar } \\
2014- \\
2015\end{array}$} & 3 & $9.44 \pm 0.05$ & 0.3 & 05 & - & 0.68 & 0.68 & - & 0.65 & 0.30 & 0.97 & $1.019 \pm 0.002$ \\
\hline & 4 & $9.21 \pm 0.03$ & 0.1 & 0.5 & 0.2 & 0.59 & 0.68 & 0.60 & 0.31 & 0.35 & 0.98 & $1.021 \pm 0.002$ \\
\hline & 5 & $8.68 \pm 0.07$ & 0.3 & 0.5 & 0.1 & 0.73 & 0.67 & 0.60 & 0.20 & 0.37 & 0.98 & $1.020 \pm 0.002$ \\
\hline \multirow{3}{*}{$\begin{array}{l}\text { Apr-May } \\
2015\end{array}$} & 3 & $4.33 \pm 0.09$ & 0.5 & 0.5 & - & 0.74 & 0.45 & - & 0.40 & 0.26 & 0.95 & $1.023 \pm 0.004$ \\
\hline & 4 & $4.10 \pm 0.05$ & 0.3 & 0.5 & - & 0.70 & 0.43 & & 0.39 & 0.17 & 0.96 & $1.025 \pm 0.004$ \\
\hline & 5 & $3.70 \pm 0.05$ & 0.1 & 0.5 & 0.3 & 0.88 & 0.5 & 0.4 & 0.26 & 0.26 & 0.96 & $1.032 \pm 0.004$ \\
\hline \multirow{2}{*}{ B } & \multirow{2}{*}{$\mathrm{n}$} & \multirow{2}{*}{ Q/Qexp } & \multirow{2}{*}{$\begin{array}{c}\text { a-value } \\
\text { COA }\end{array}$} & \multirow{2}{*}{$\begin{array}{c}\text { a-value } \\
\text { HOA }\end{array}$} & \multirow{2}{*}{$\begin{array}{l}\text { a-value } \\
\text { BBOA }\end{array}$} & \multirow{2}{*}{$\begin{array}{c}\text { COA vs. } \\
\mathrm{m} / \mathrm{z} 55\end{array}$} & \multirow{2}{*}{$\begin{array}{l}\text { HOA } \\
\text { vs. BC }\end{array}$} & \multirow{2}{*}{$\begin{array}{c}\text { BBOA } \\
\text { vs. } \\
\mathrm{m} / \mathrm{z} 60\end{array}$} & LO-OO & MO-OO & OA m & Vs. OA app. \\
\hline & & & & & & & & & $\begin{array}{l}\text { A vs. } \\
\mathrm{NO}_{3}{ }^{-}\end{array}$ & $\begin{array}{l}\text { A vs. } \\
\mathrm{SO}_{4}^{2-}\end{array}$ & $R^{2}$ & Slope \\
\hline & 3 & $1.81 \pm 0.001$ & 0.1 & 0.5 & - & 0.83 & 0.51 & - & 0.60 & 0.15 & 0.96 & $1.00+0.14$ \\
\hline se & 4 & $1.053 \pm 0.012$ & 0.1 & 0.5 & - & 0.80 & 0.52 & - & 0.59 & 0.33 & 0.96 & $1.01 x+0.09$ \\
\hline & 5 & $0.977 \pm 0.006$ & 0.1 & 0.3 & 0.5 & 0.61 & 0.65 & 0.28 & 0.53 & 0.18 & 0.96 & $0.96 x+0.09$ \\
\hline & 4 & $0.873 \pm 0.003$ & 0.3 & 0.5 & 0.2 & 0.68 & 0.78 & 0.87 & 0.80 & 0.46 & 0.97 & $1.013 x+0.07$ \\
\hline Mar & 5 & $0.808 \pm 0.004$ & 0.2 & 0.5 & 0.1 & 0.72 & 0.61 & 0.91 & 0.32 & 0.47 & 0.97 & $1.010 x+0.032$ \\
\hline $\begin{array}{l}201 /- \\
2018\end{array}$ & 6 & $0.765 \pm 0.002$ & 0.3 & 0.5 & 0.3 & 0.70 & 0.78 & 0.91 & 0.41 & 0.47 & 0.97 & $1.01 x+0.12$ \\
\hline Apr- & 3 & $1.076 \pm 0.000$ & 0.1 & 0.5 & - & 0.78 & 0.65 & - & 0.51 & 0.18 & 0.97 & $1.0 x+0.2$ \\
\hline May & 4 & $0.893 \pm 0.006$ & 0.4 & 0.5 & - & 0.76 & 0.57 & - & 0.42 & 0.32 & 0.97 & $0.90 x+0.13$ \\
\hline & 5 & $0.778 \pm 0.006$ & 0.3 & 0.2 & 0.3 & 0.69 & 0.56 & 0.57 & 0.42 & 0.31 & 0.97 & $1.00 x+0.08$ \\
\hline & 3 & $0.957 \pm 0.013$ & 0.5 & 0.5 & - & 0.69 & 0.31 & - & 0.04 & 0.05 & 0.97 & $1.00 x+0.15$ \\
\hline Jun-Aug & 4 & $0.869 \pm 0.008$ & 0.5 & 0.5 & - & 0.76 & 0.44 & - & 0.1 & 0.08 & 0.96 & $1.00 x+0.14$ \\
\hline & 5 & $0.809 \pm 0.001$ & 0.5 & 0.4 & - & 0.76 & 0.47 & - & 0.14 & 0.07 & 0.96 & $1.12 x+0.15$ \\
\hline & 3 & $1.493 \pm 0.008$ & 0.5 & 0.5 & - & 0.73 & 0.74 & - & 0.25 & 0.27 & 0.95 & $1.02 x+0.03$ \\
\hline Sep-Oct & 4 & $1.273 \pm 0.001$ & 0.3 & 0.5 & - & 0.74 & 0.74 & - & 0.23 & 0.21 & 0.97 & $1.02 x-0.02$ \\
\hline & 5 & $0.923 \pm 0.003$ & 0.3 & 0.2 & 0.1 & 0.73 & 0.73 & 0.55 & 0.17 & 0.16 & 0.97 & $1.03 x-0.04$ \\
\hline
\end{tabular}


Table S2. Correlations of factors mass spectra with external anchor mass spectra (a), and factors time series with external markers (b).

\begin{tabular}{|c|c|c|c|c|c|c|c|c|c|c|}
\hline & \multirow[t]{2}{*}{ (a) } & \multicolumn{4}{|c|}{ Crippa et al., 2013, *Ng et al., 2010} & \multicolumn{5}{|c|}{ Mohr et al., 2012} \\
\hline & & COA & HOA & BBOA* & OOA & COA & HOA & BBOA & SV-OOA & LV-OOA \\
\hline \multirow{5}{*}{ A } & COA & 0.96 & 0.87 & 0.74 & 0.35 & 0.92 & 0.76 & 0.76 & 0.71 & 0.19 \\
\hline & HOA & 0.77 & 0.96 & 0.62 & 0.34 & 0.71 & 0.93 & 0.62 & 0.61 & 0.14 \\
\hline & BBOA & 0.25 & 0.13 & 0.67 & 0.36 & 0.38 & 0.08 & 0.35 & 0.34 & 0.27 \\
\hline & LO-OOA & 0.13 & 0.06 & 0.31 & 0.95 & 0.27 & 0.03 & 0.28 & 0.66 & 0.97 \\
\hline & MO-OOA & 0.10 & 0.05 & 0.25 & 0.93 & 0.22 & 0.02 & 0.24 & .65 & 0.95 \\
\hline \multirow{5}{*}{ B } & COA & 0.98 & 0.71 & 0.66 & 0.27 & 0.86 & 0.58 & 0.66 & 0.65 & 0.16 \\
\hline & HOA & 0.79 & 0.99 & 0.61 & 0.19 & 0.73 & 0.94 & 0.65 & 0.55 & 0.10 \\
\hline & BBOA & 0.62 & 0.50 & 0.99 & 0.40 & 0.77 & 0.40 & 0.72 & 0.65 & 0.33 \\
\hline & LO-OOA & 0.39 & 0.25 & 0.70 & 0.79 & 0.64 & 0.16 & 0.66 & 0.75 & 0.72 \\
\hline & MO-OOA & 0.07 & 0.02 & 0.03 & 0.89 & 0.17 & 0.05 & 0.16 & 0.56 & 0.94 \\
\hline
\end{tabular}

\begin{tabular}{|c|c|c|c|c|c|c|c|c|c|c|}
\hline (b) & $\begin{array}{c}\text { Period } \\
\text { A }\end{array}$ & $\begin{array}{l}\text { Apr- } \\
\text { May } \\
2015\end{array}$ & $\begin{array}{l}\text { Jun- } \\
\text { Aug } \\
2014\end{array}$ & $\begin{array}{c}\text { Sep - } \\
\text { Oct } \\
2014\end{array}$ & $\begin{array}{c}\text { Nov- } \\
\text { Mar } \\
2014- \\
2015\end{array}$ & $\begin{array}{c}\text { Period } \\
\text { B }\end{array}$ & $\begin{array}{c}\text { Ap- } \\
\text { May } \\
2018\end{array}$ & $\begin{array}{c}\text { Jun- } \\
\text { Aug } \\
2018\end{array}$ & $\begin{array}{c}\text { Sep- } \\
\text { Oct } \\
2018\end{array}$ & $\begin{array}{c}\text { Nov- } \\
\text { Mar } \\
2017- \\
2018\end{array}$ \\
\hline COA vs. m/z 55 & 0.58 & 0.69 & 0.76 & 0.35 & 0.63 & 0.71 & 0.76 & 0.76 & 0.74 & 0.72 \\
\hline COA vs. HOA & 0.20 & 0.32 & 0.24 & 0.02 & 0.26 & 0.31 & 0.32 & 0.32 & 0.28 & 0.33 \\
\hline HOA vs. BC & 0.62 & 0.42 & 0.40 & 0.70 & 0.63 & 0.68 & 0.57 & 0.57 & 0.74 & 0.68 \\
\hline HOA vs. NO & 0.44 & 0.36 & 0.16 & 0.53 & 0.42 & 0.63 & 0.41 & 0.41 & 0.68 & 0.76 \\
\hline $\mathrm{HOA}$ vs. $\mathrm{NO}_{2}$ & 0.49 & 0.33 & 0.39 & 0.51 & 0.49 & 0.55 & 0.51 & 0.51 & 0.70 & 0.75 \\
\hline HOA vs. $\mathrm{NO}_{\mathrm{x}}$ & 0.44 & 0.40 & 0.29 & 0.45 & 0.44 & 0.65 & 0.51 & 0.51 & 0.70 & 0.75 \\
\hline LO-OOA vs. $\mathrm{m} / \mathrm{z} 43$ & 0.51 & 0.48 & 0.25 & 0.60 & - & 0.41 & 0.86 & 0.86 & 0.84 & 0.66 \\
\hline LO-OOA vs. $\mathrm{NO}_{3}{ }^{-}$ & 0.29 & 0.39 & 0.74 & 0.34 & - & 0.60 & 0.40 & 0.68 & 0.75 & 0.58 \\
\hline MO-OOA vs. m/z 44 & 0.60 & 0.40 & 0.68 & 0.75 & 0.58 & 0.69 & 0.84 & 0.84 & 0.96 & 0.96 \\
\hline MO-OOA vs. $\mathrm{SO}_{4}^{2-}$ & 0.02 & 0.09 & 0.13 & 0.03 & 0.03 & 0.10 & 0.32 & 0.32 & 0.31 & 0.47 \\
\hline BBOA vs. $\mathrm{m} / \mathrm{z} 60$ & 0.60 & - & - & - & 0.60 & 0.91 & - & - & - & 0.91 \\
\hline BBOA vs. $\mathrm{m} / \mathrm{z} 73$ & 0.55 & - & - & - & 0.55 & 0.61 & - & - & - & 0.61 \\
\hline
\end{tabular}


(a)
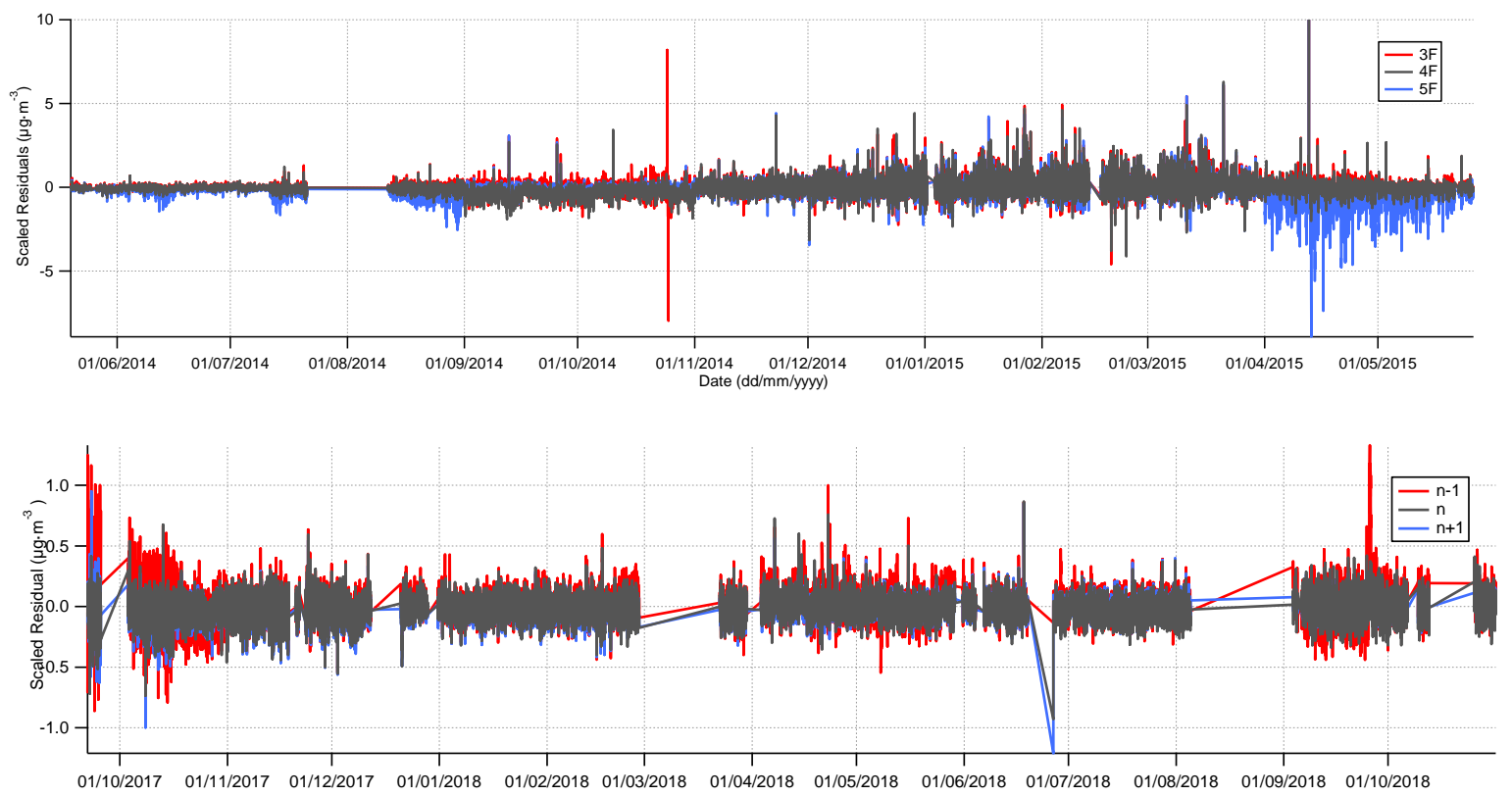

(b)

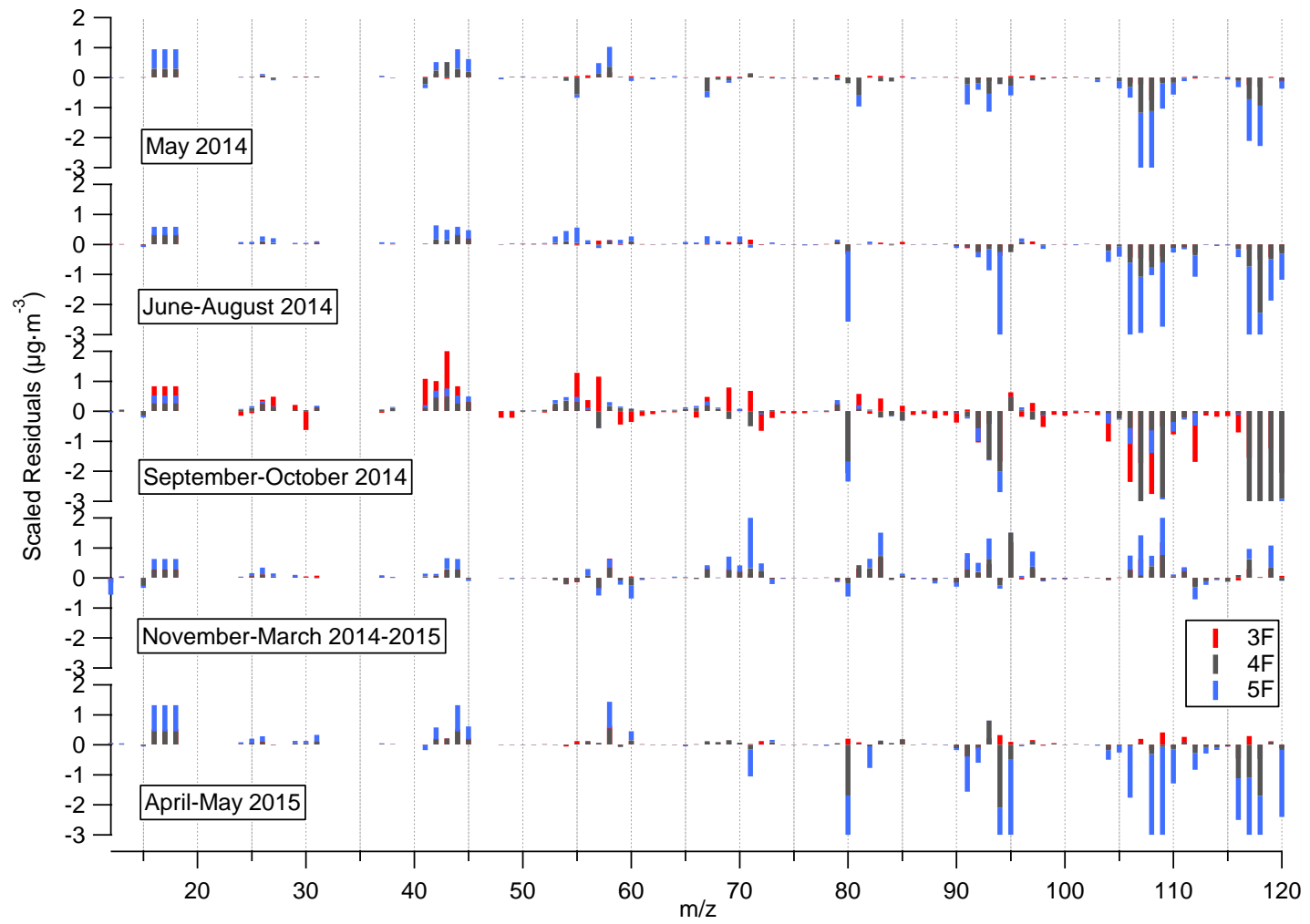




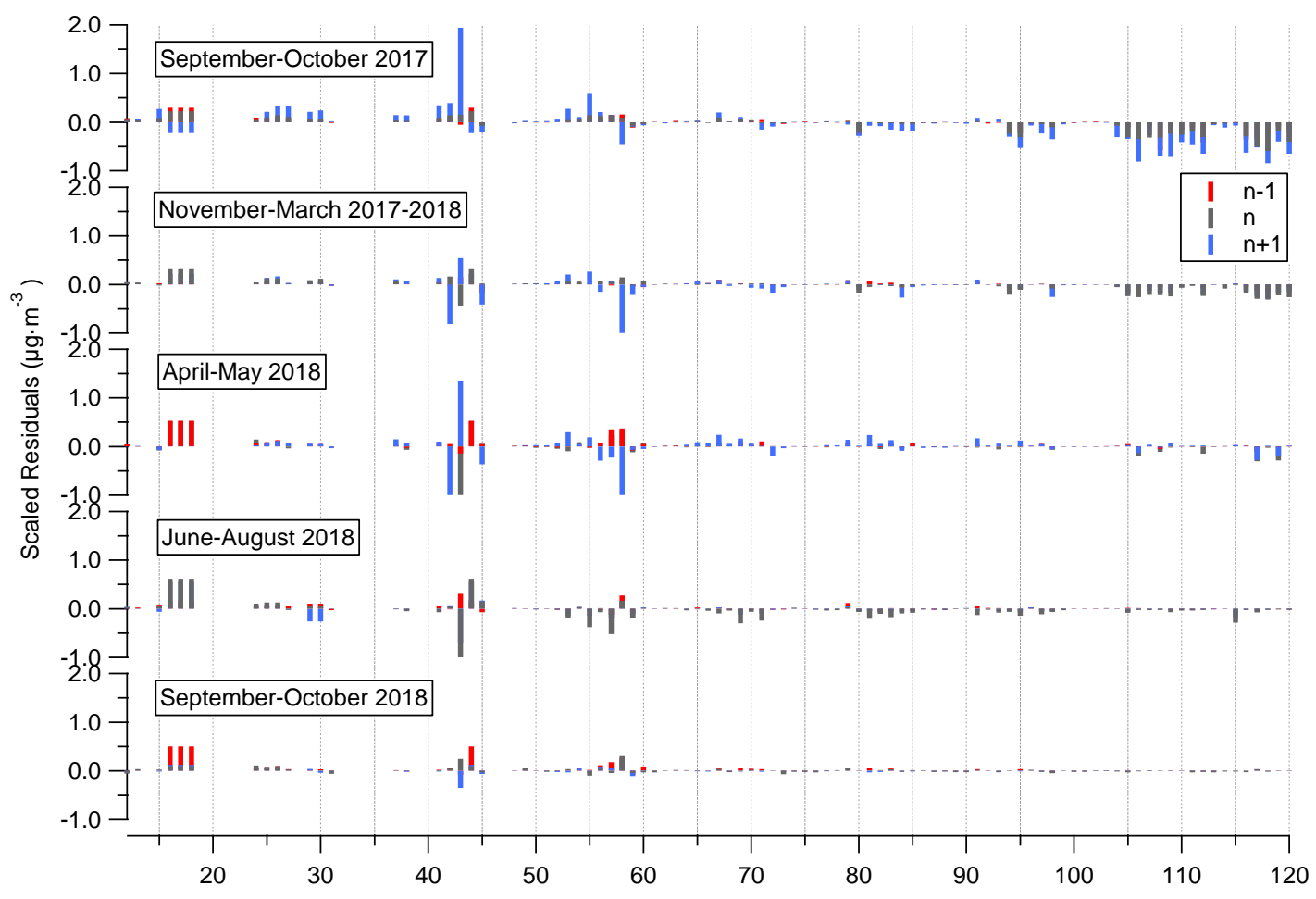

Figure S 3. Scaled residuals (a) time series (b) mass spectra for 3, 4, 5 factors in period $A$ and for $n-1, n$ and $n+1$ factors in Period $B$ being $n$ the number of factors of the chosen solution.
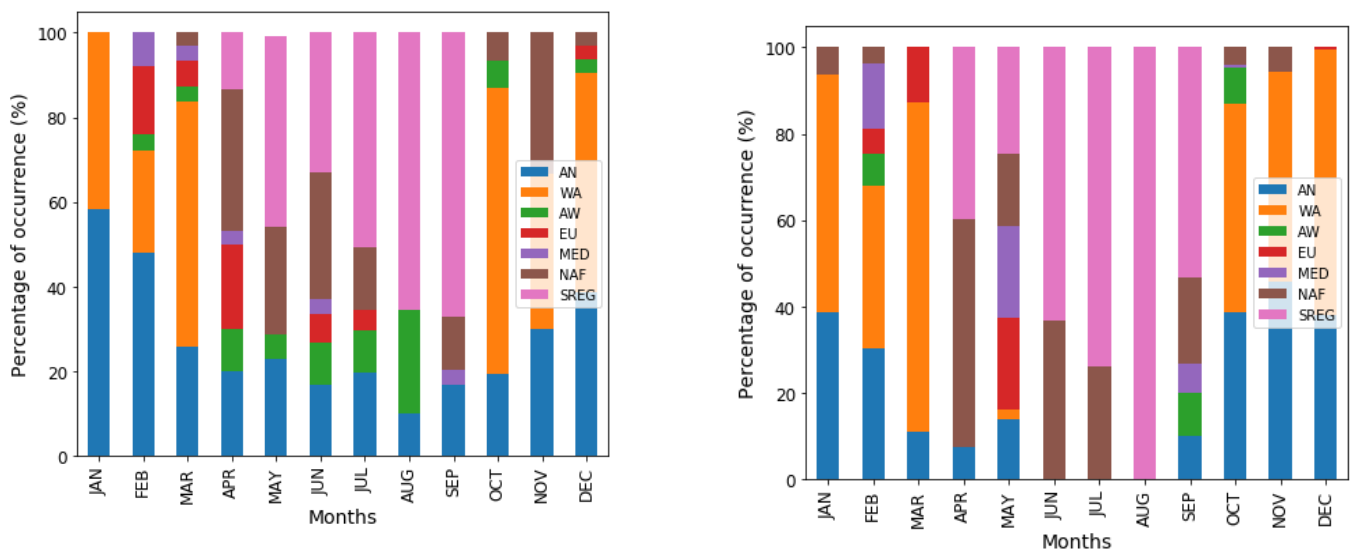

Figure S 4. Relative frequency of occurrence of episodes sorted by month for periods A (left) and B (right).

Table S 3. Comparison between i. total $\mathrm{PM}_{1}$ from the sum of ACSM components (NR-PMi) and BC concentrations and co-located measurements of PM from SMPS and ii. ACSM species concentration vs. 24-h samples concentrations. Fit parameters correspond to least orthogonal distance regression method.

\begin{tabular}{|l|l|l|l|l|l|l|l|}
\hline \multicolumn{2}{|l|}{ (i) } & \multicolumn{2}{l|}{ A } & \multicolumn{3}{l|}{ B } \\
\hline$y$ & $x$ & $R^{2}$ & Slope & Intercept & $R^{2}$ & Slope & Intercept \\
\hline NR-PM $1+B C$ & SMPS (Mass) & 0.61 & $1.001 \pm 0.006$ & $0.37 \pm 0.07$ & 0.72 & $1.177 \pm 0.006$ & $-0.47 \pm 0.05$ \\
\hline
\end{tabular}




\begin{tabular}{|l|l|l|l|l|l|l|l|}
\hline \multicolumn{2}{|l|}{ (ii) } & \multicolumn{2}{l}{ A } & \multicolumn{2}{l|}{ B } \\
\hline$y$ & $x$ & $R^{2}$ & Slope & Intercept & $R^{2}$ & Slope & Intercept \\
\hline $\mathrm{SO}_{4}{ }^{2-} \mathrm{ACSM}$ & $\mathrm{SO}_{4}{ }^{2-}$ off-line & 0.88 & $1.24 \pm 0.05$ & $-0.31 \pm 0.10$ & 0.93 & $1.05 \pm 0.04$ & $0.01 \pm 0.08$ \\
\hline $\mathrm{NO}_{3}-\mathrm{ACSM}$ & $\mathrm{NO}_{3}$ - off-line & 0.84 & $1.9 \pm 0.1$ & $-0.12 \pm 0.09$ & 0.86 & $1.76 \pm 0.09$ & $0.32 \pm 0.09$ \\
\hline $\mathrm{NH}_{4}{ }^{+} \mathrm{ACSM}$ & $\mathrm{NH} 4^{+}$off-line & 0.85 & $1.73 \pm 0.08$ & $-0.27 \pm 0.07$ & 0.71 & $1.68 \pm 0.13$ & $0.05 \pm 0.09$ \\
\hline OA ACSM & OC off-line & 0.73 & $2.69 \pm 0.17$ & $-0.9 \pm 0.4$ & 0.86 & $2.94 \pm 0.17$ & $-1.4 \pm 0.3$ \\
\hline
\end{tabular}

(a)
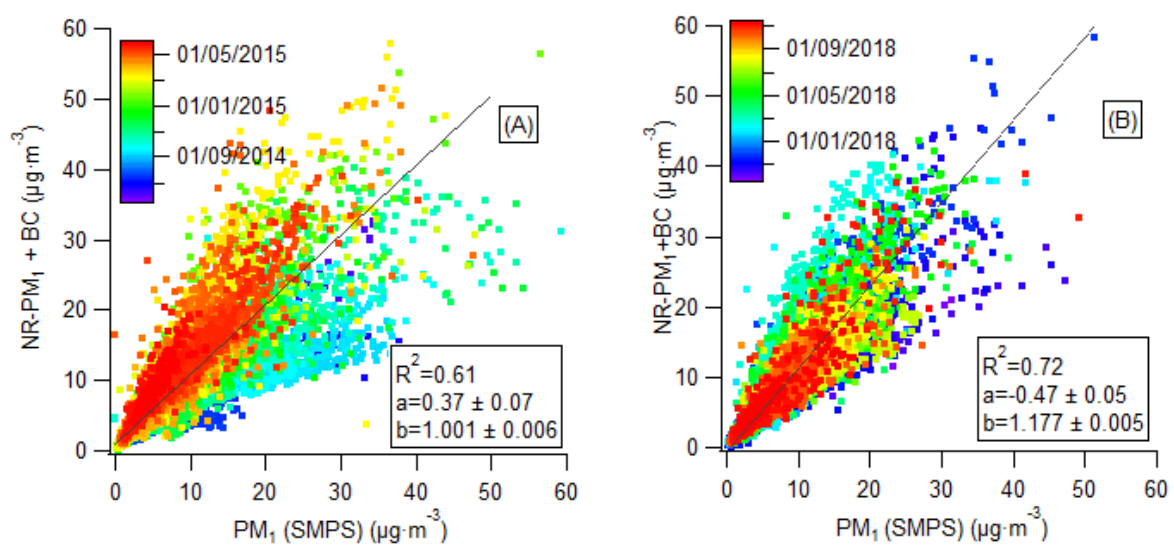

(b)
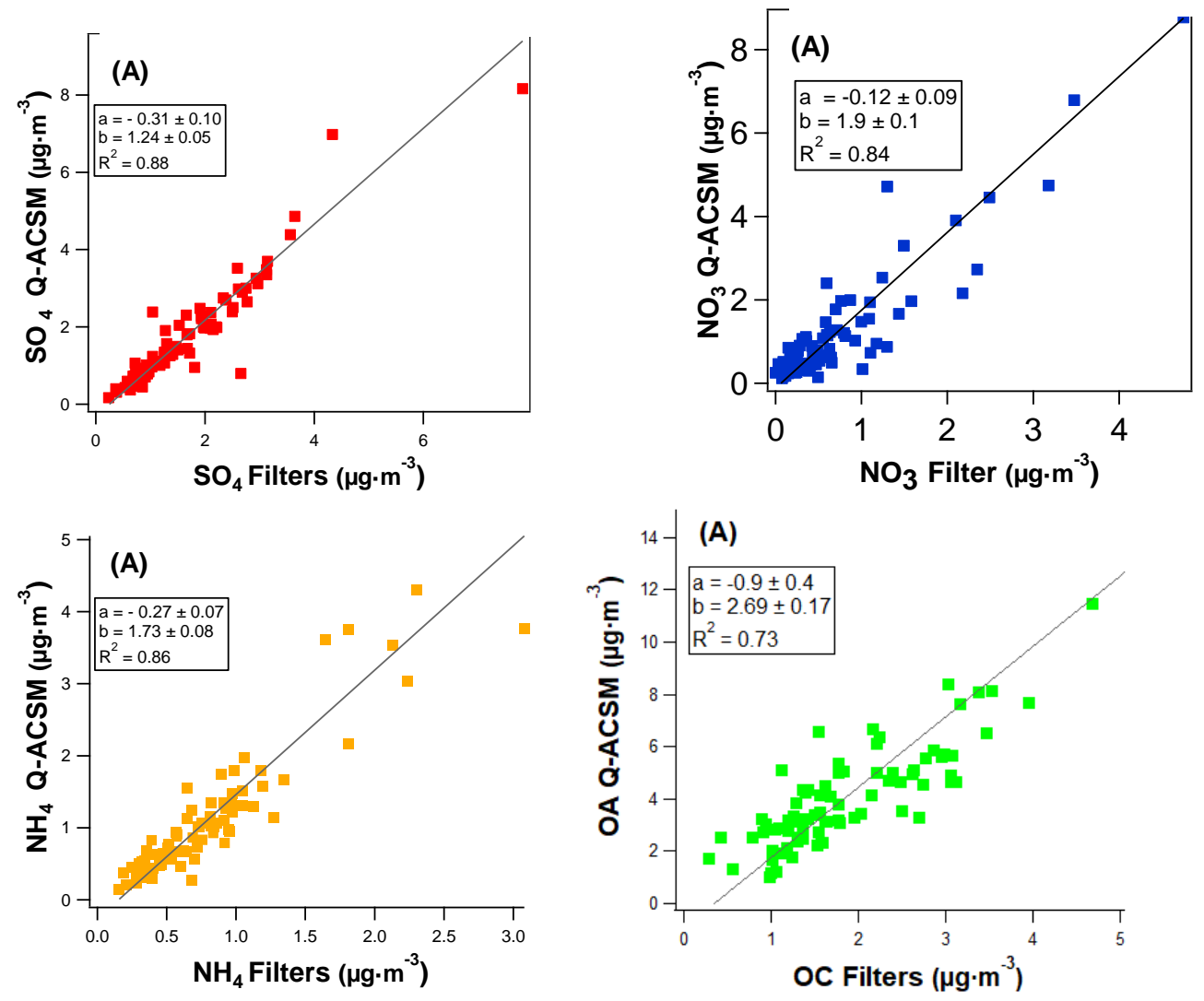

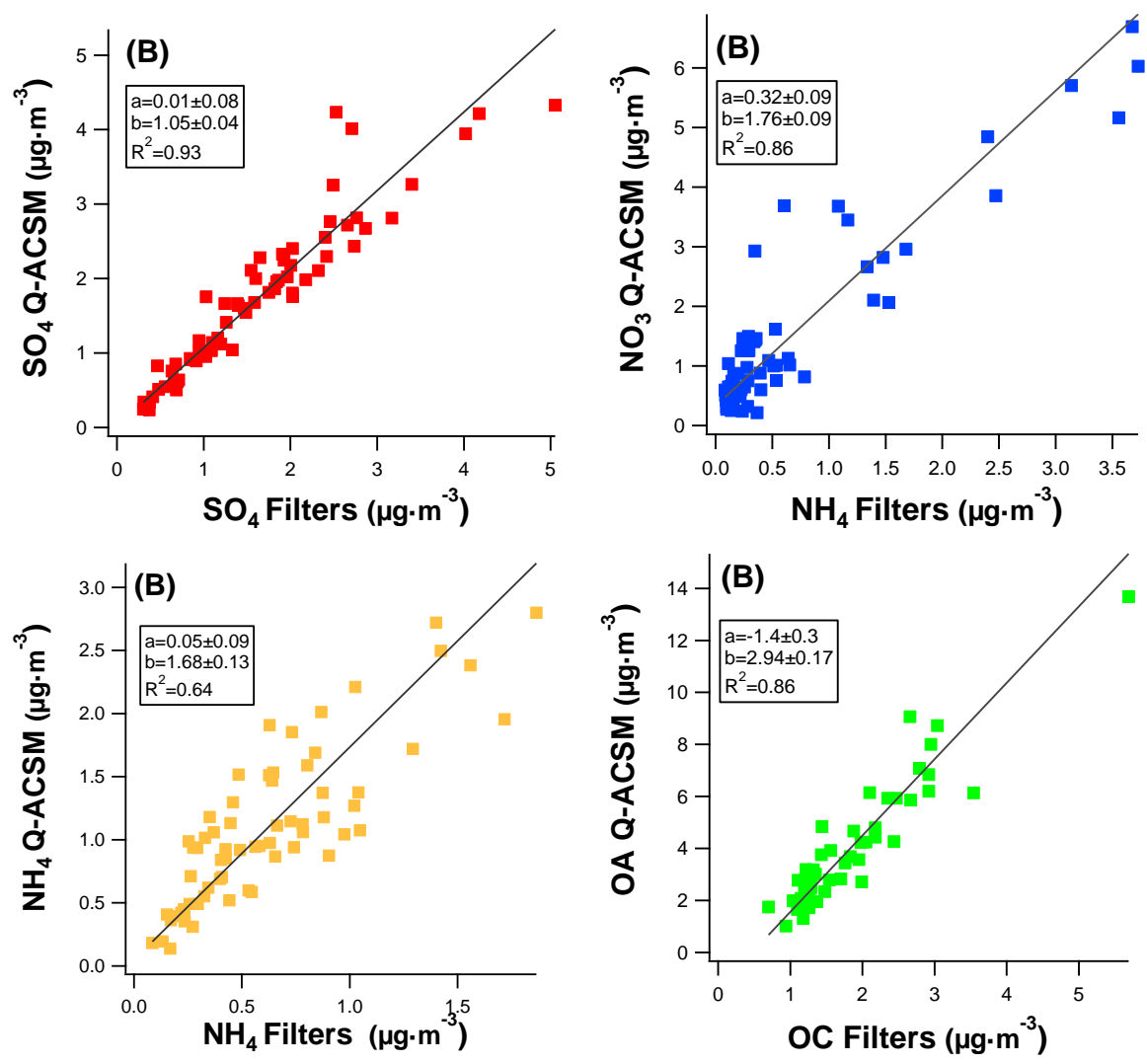

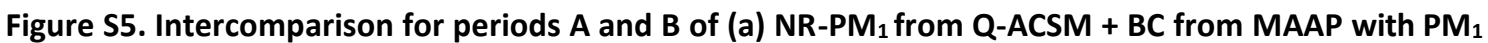
measurements from SMPS. (b) NR-PM1 species from Q-ACSM with $\mathrm{SO}_{4}{ }^{2-}, \mathrm{NO}_{3}{ }^{-}, \mathrm{NH}_{4}{ }^{+}$and $\mathrm{OC}$ from filters, respectively.

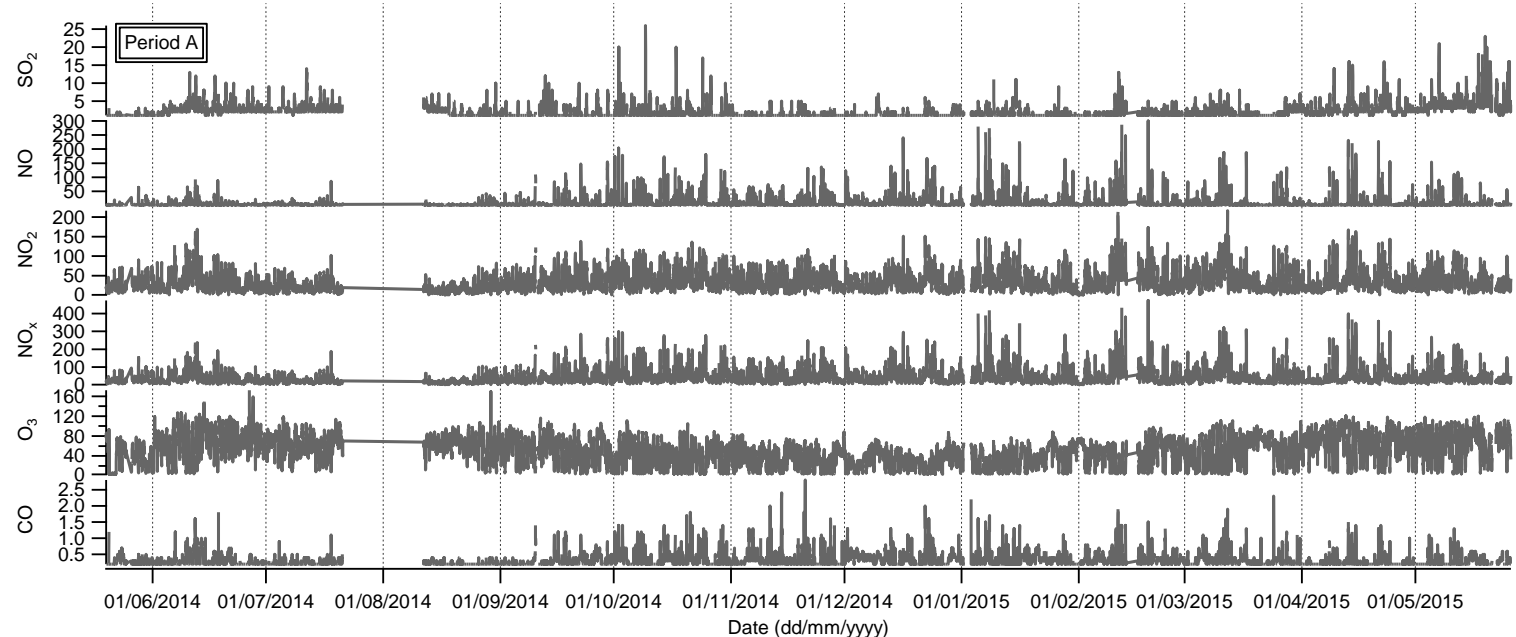


is 250

$2 \begin{aligned} & 400-1 \\ & 200 \\ & 100 \\ & 100\end{aligned}$

$\stackrel{1}{80}_{40}^{160} \mathrm{Z}$

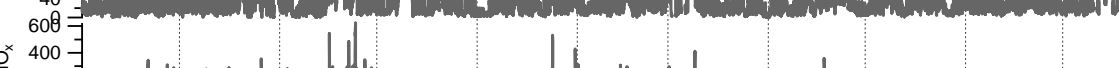

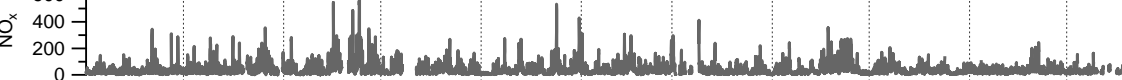

120 年

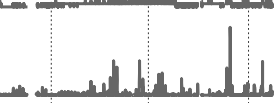

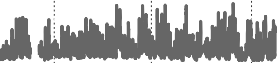
40 拓,

O

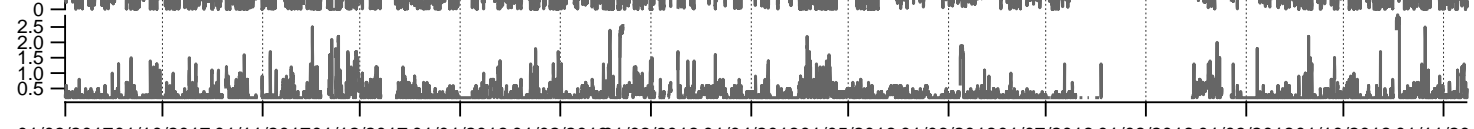
01/09/201701/10/2017 01/11/201701/12/2017 01/01/201801/02/201ه1/03/2018 01/04/201801/05/2018 01/06/201801/07/2018 01/08/2018 01/09/201801/10/2018 01/11/2018 Date $(\mathrm{dd} / \mathrm{mm} / \mathrm{yyyy})$

Figure S 6. Time series of co-located gases measurements for period A and B.
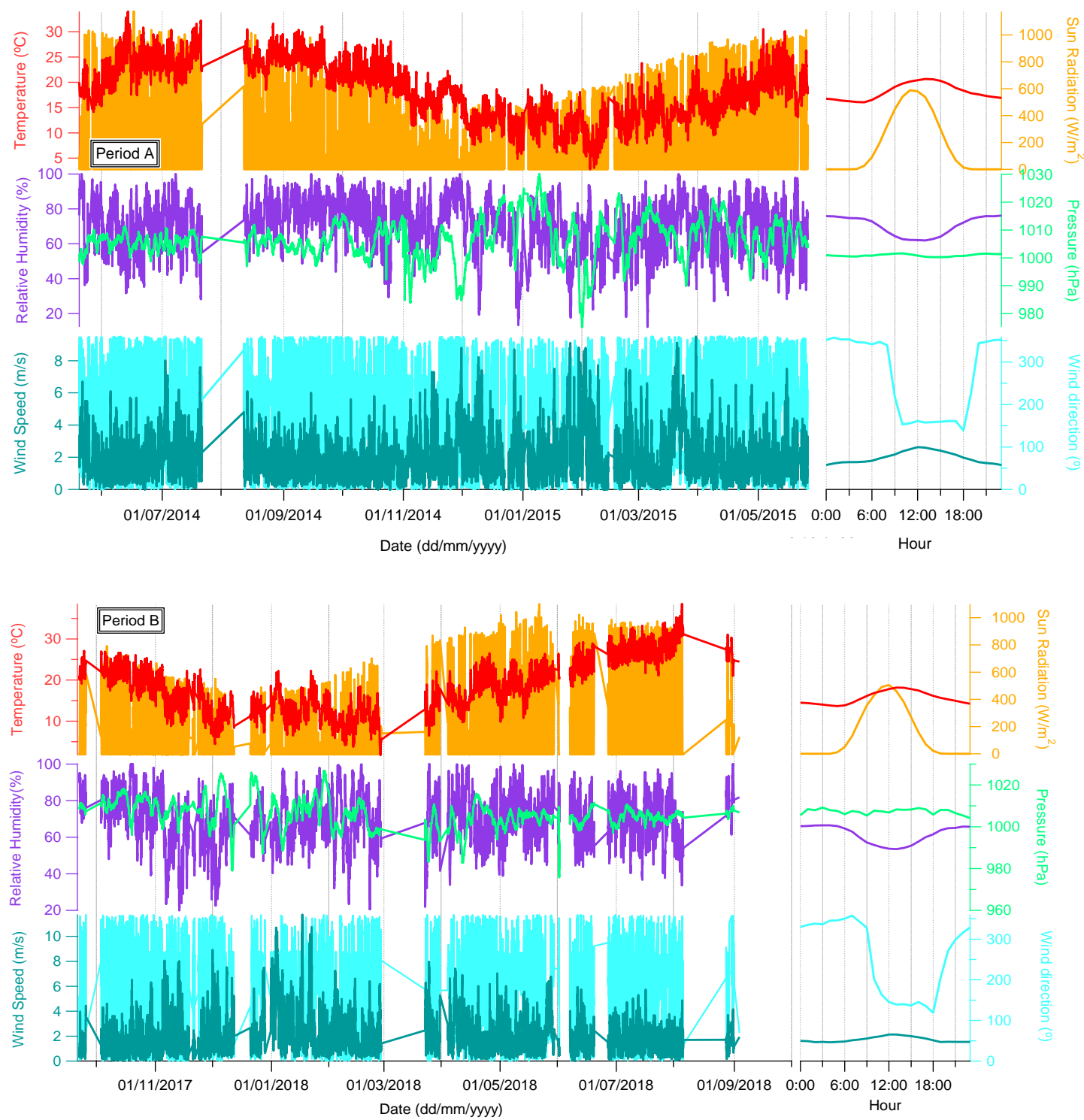

Figure S 7. Time series and diel patterns of meteorological variables for periods A (top) and B (bottom). 
Table S4. Period-average mean, standard deviation and percentual change of variables under study for periods $A$ and $B$.

\begin{tabular}{|c|c|c|c|c|c|c|c|}
\hline & & & \multicolumn{2}{|c|}{ Period A } & \multicolumn{2}{|c|}{ Period B } & \multirow{2}{*}{$\begin{array}{c}\text { Change } A \text { to } B \\
\text { (\%) }\end{array}$} \\
\hline & Variables & Units & Average & $\begin{array}{l}\text { Standard } \\
\text { Deviation }\end{array}$ & Average & $\begin{array}{l}\text { Standard } \\
\text { Deviation }\end{array}$ & \\
\hline \multirow{6}{*}{$\begin{array}{c}\text { Meteorological } \\
\text { parameters }\end{array}$} & Temperature & ${ }^{\circ} \mathrm{C}$ & 18.2 & 5.7 & 18.4 & 6.8 & $+1 \%$ \\
\hline & Relative Humidity & $\%$ & 70.7 & 15.3 & 70.2 & 14.8 & $-0.7 \%$ \\
\hline & Pressure & $\mathrm{hPa}$ & 1006.5 & 7.3 & 1005.5 & 7.32 & $-0.1 \%$ \\
\hline & Sun Radiation & $w \cdot m^{-2}$ & 180.0 & 266.0 & 178.9 & 268.5 & $-0.6 \%$ \\
\hline & Wind Speed & $\mathrm{m} \cdot \mathrm{s}^{-1}$ & 2.0 & 1.4 & 2.0 & 1.5 & $0 \%$ \\
\hline & Wind Direction & $\circ$ & 170.0 & 102.0 & 200.4 & 104.8 & $+30.4^{\circ}$ \\
\hline \multirow{5}{*}{$\begin{array}{l}\text { Gas-phase } \\
\text { pollutants }\end{array}$} & $\mathrm{O}_{3}$ & $\mu g \cdot m^{-3}$ & 52.4 & 28.0 & 47.6 & 23.8 & $-9 \%$ \\
\hline & Co & $\mu g \cdot m^{-3}$ & 0.3 & 0.2 & 0.3 & 0.3 & $0 \%$ \\
\hline & $\mathrm{SO}_{2}$ & $\mu g \cdot m^{-3}$ & 2.0 & 1.7 & 1.7 & 1.4 & $-15 \%$ \\
\hline & NO & $\mu g \cdot m^{-3}$ & 8.3 & 19.2 & 8.5 & 1.2 & $+2 \%$ \\
\hline & $\mathrm{NO}_{2}$ & $\mu g \cdot m^{-3}$ & 32.3 & 24.8 & 30.6 & 21.3 & $-5 \%$ \\
\hline \multirow{5}{*}{$\begin{array}{l}\text { NR-PM } \text { A ACSM }_{1} \\
\text { Species }\end{array}$} & OA & $\mu g \cdot m^{-3}$ & 4.2 & 2.8 & 4.0 & 2.8 & $-5 \%$ \\
\hline & $\mathrm{SO}_{4}{ }^{2-}$ & $\mu g \cdot m^{-3}$ & 1.9 & 1.5 & 1.5 & 1.2 & $-21 \%$ \\
\hline & $\mathrm{NO}_{3}^{-}$ & $\mu g \cdot m^{-3}$ & 1.3 & 1.7 & 1.4 & 1.9 & $8 \%$ \\
\hline & $\mathrm{NH}_{4}^{+}$ & $\mu \mathrm{g} \cdot \mathrm{m}^{-3}$ & 1.1 & 0.9 & 1.0 & 0.8 & $-9 \%$ \\
\hline & $\mathrm{Cl}^{-}$ & $\mu g \cdot m^{-3}$ & 0.05 & 0.08 & 0.06 & 0.08 & $+20 \%$ \\
\hline MAAP & BC & $\mu g \cdot m^{-3}$ & 1.7 & 1.6 & 1.4 & 1.4 & $-18 \%$ \\
\hline ACSM + MAAP & $\mathrm{PM}_{1}$ & $\mu g \cdot m^{-3}$ & 10.1 & 6.7 & 9.6 & 6.6 & $-5 \%$ \\
\hline OPC & $\mathrm{PM}_{1}$ & $\mu g \cdot m^{-3}$ & 8.3 & 5.4 & 9.1 & 5.0 & $+9 \%$ \\
\hline SMPS & $\mathrm{PM}_{1}$ & $\mu g \cdot m^{-3}$ & 9.8 & 7.0 & 8.5 & 5.3 & $-13 \%$ \\
\hline Filters & $\mathrm{PM}_{1}$ & $\mu \mathrm{g} \cdot \mathrm{m}^{-3}$ & 8.0 & 4.2 & 7.1 & 3.0 & $-11 \%$ \\
\hline \multirow{7}{*}{ OA contribution } & OA apportioned & $\mu \mathrm{g} \cdot \mathrm{m}^{-3}$ & 4.1 & 2.8 & 3.8 & 2.7 & $-7 \%$ \\
\hline & COA & $\mu \mathrm{g} \cdot \mathrm{m}^{-3}$ & 0.7 & 0.7 & 0.6 & 0.5 & $-14 \%$ \\
\hline & HOA & $\mu g \cdot m^{-3}$ & 0.8 & 1.1 & 0.5 & 0.8 & $-37 \%$ \\
\hline & BBOA & $\mu g \cdot m^{-3}$ & 0.3 & 0.4 & 0.2 & 0.4 & $-33 \%$ \\
\hline & LO-OOA & $\mu g \cdot m^{-3}$ & 1.5 & 1.0 & 1.1 & 1.2 & $-27 \%$ \\
\hline & MO-OOA & $\mu g \cdot m^{-3}$ & 1.4 & 1.3 & 1.7 & 1.2 & $21 \%$ \\
\hline & $\begin{array}{c}\text { LO-OOA / MO-OOA } \\
*\end{array}$ & (adim) & 1.1 & 1.6 & 1.0 & 1.6 & $-9 \%$ \\
\hline
\end{tabular}



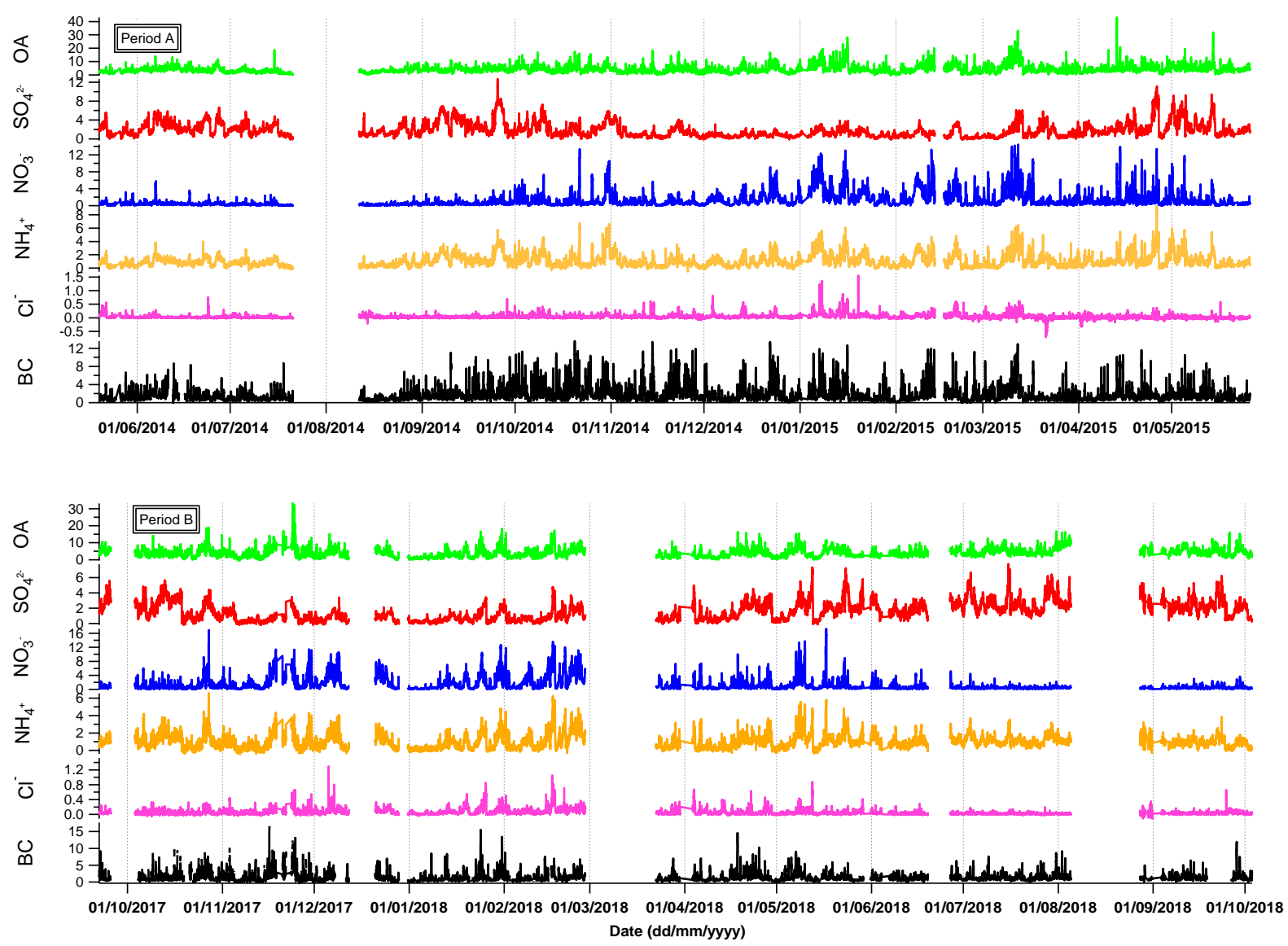

Figure S 8. Time series for NR-PM1 species and BC concentrations in periods A (top) and B (bottom).
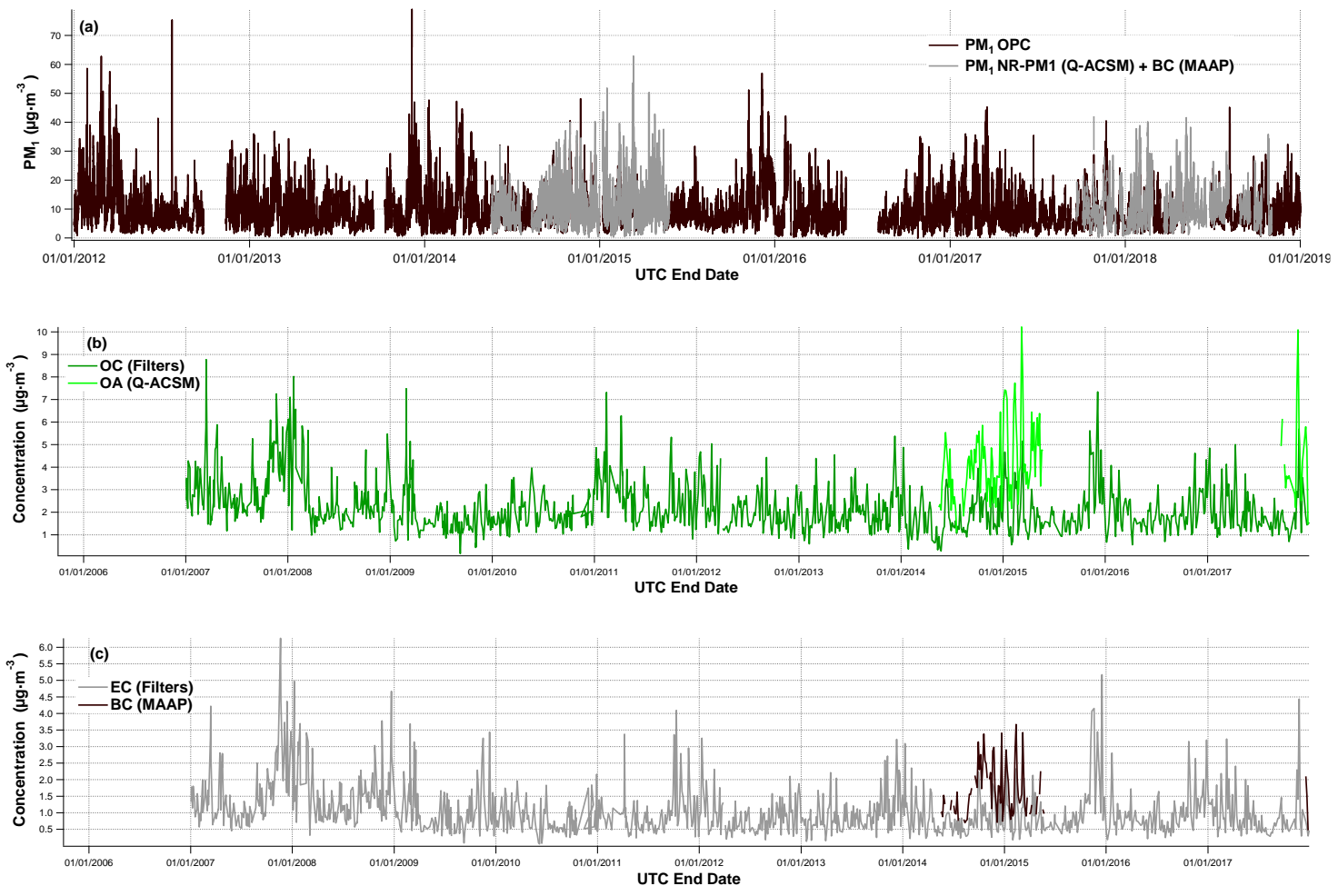

Figure S9. Figure R1. Long-term time series compared to this study two-period time series for respectively, (a) PM1 from OPC and Q-ACSM + MAAP. (b) OC from filters and OM from Q-ACSM. (c) EC from filters and BC from MAAP. 

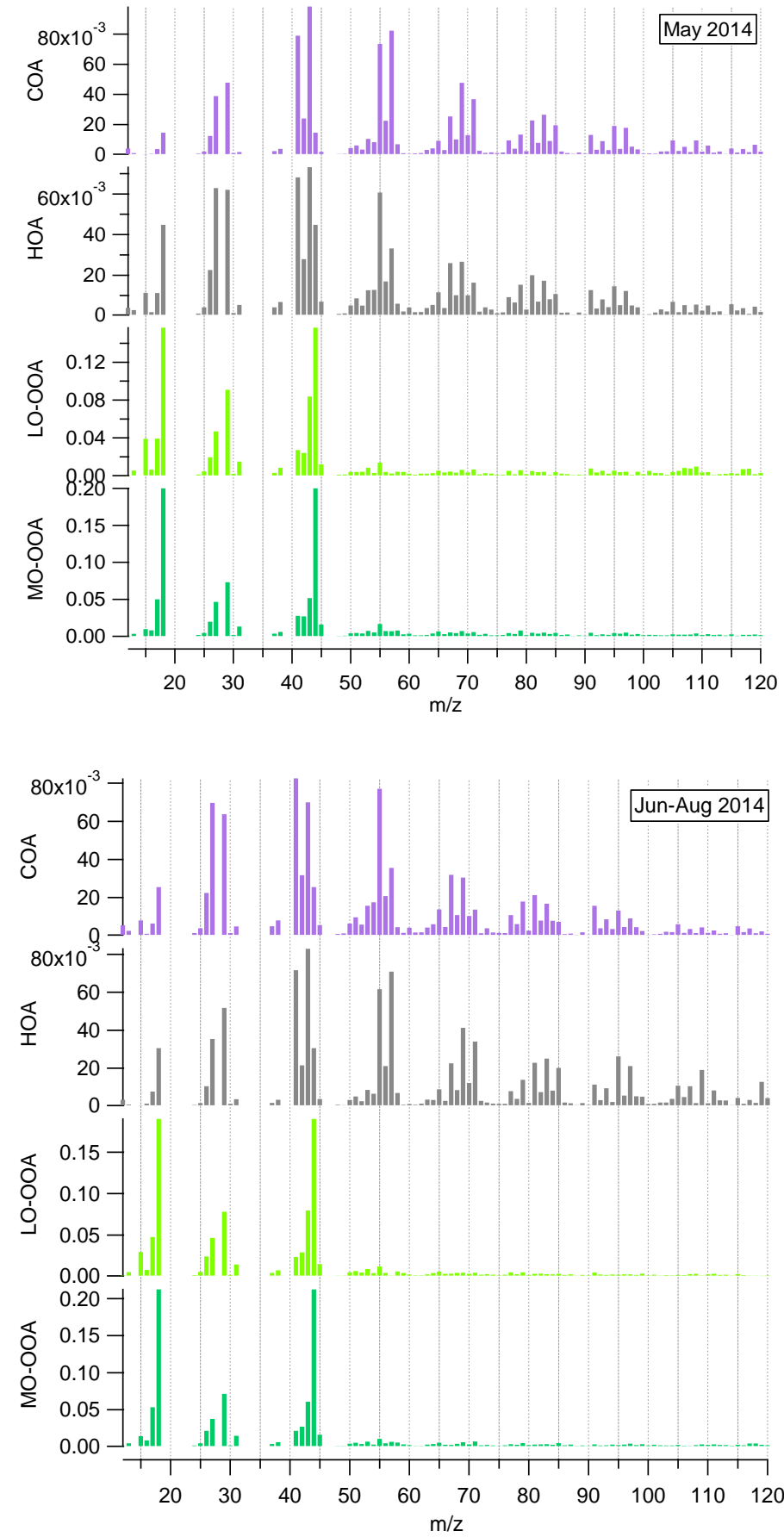

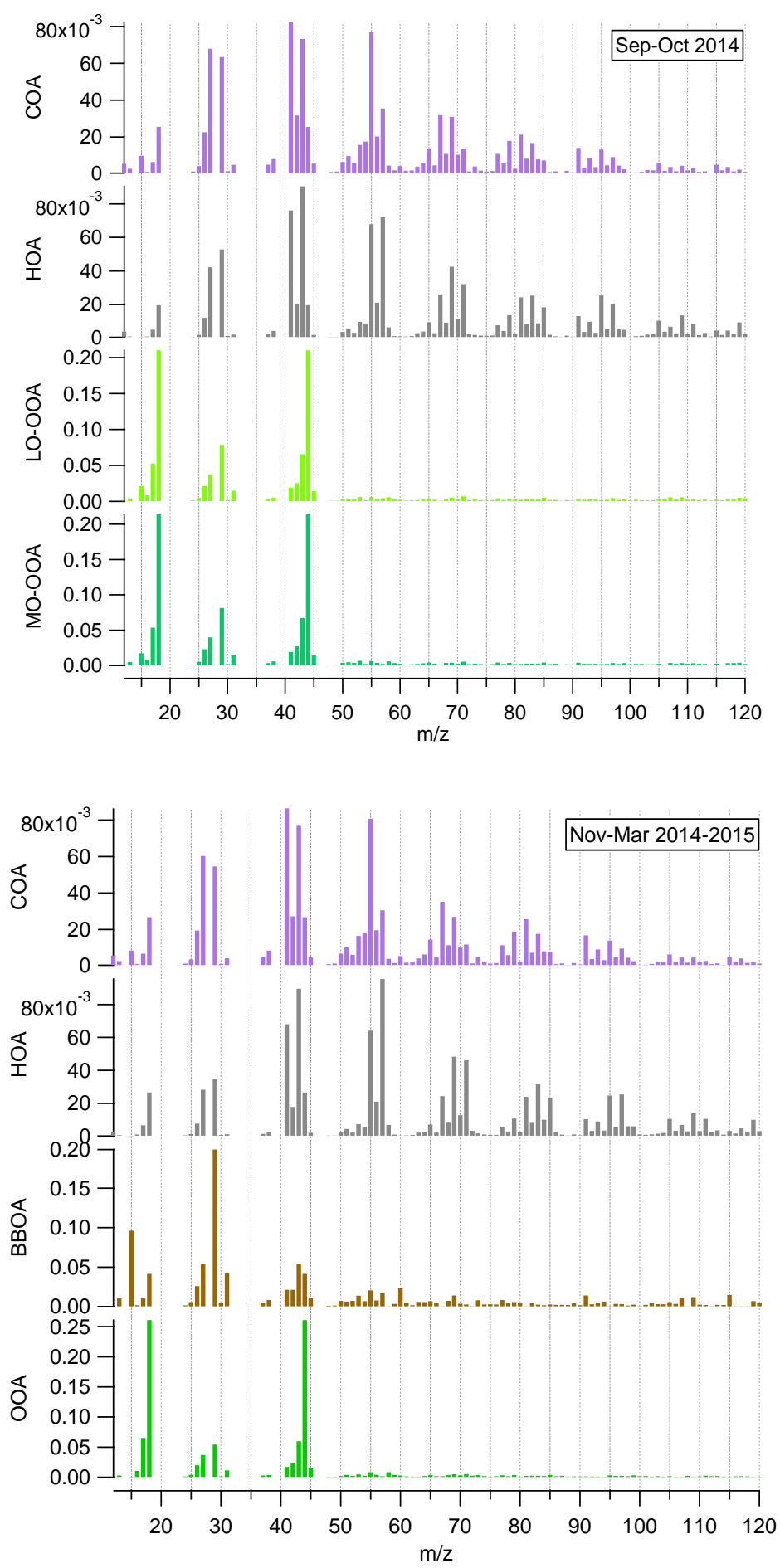

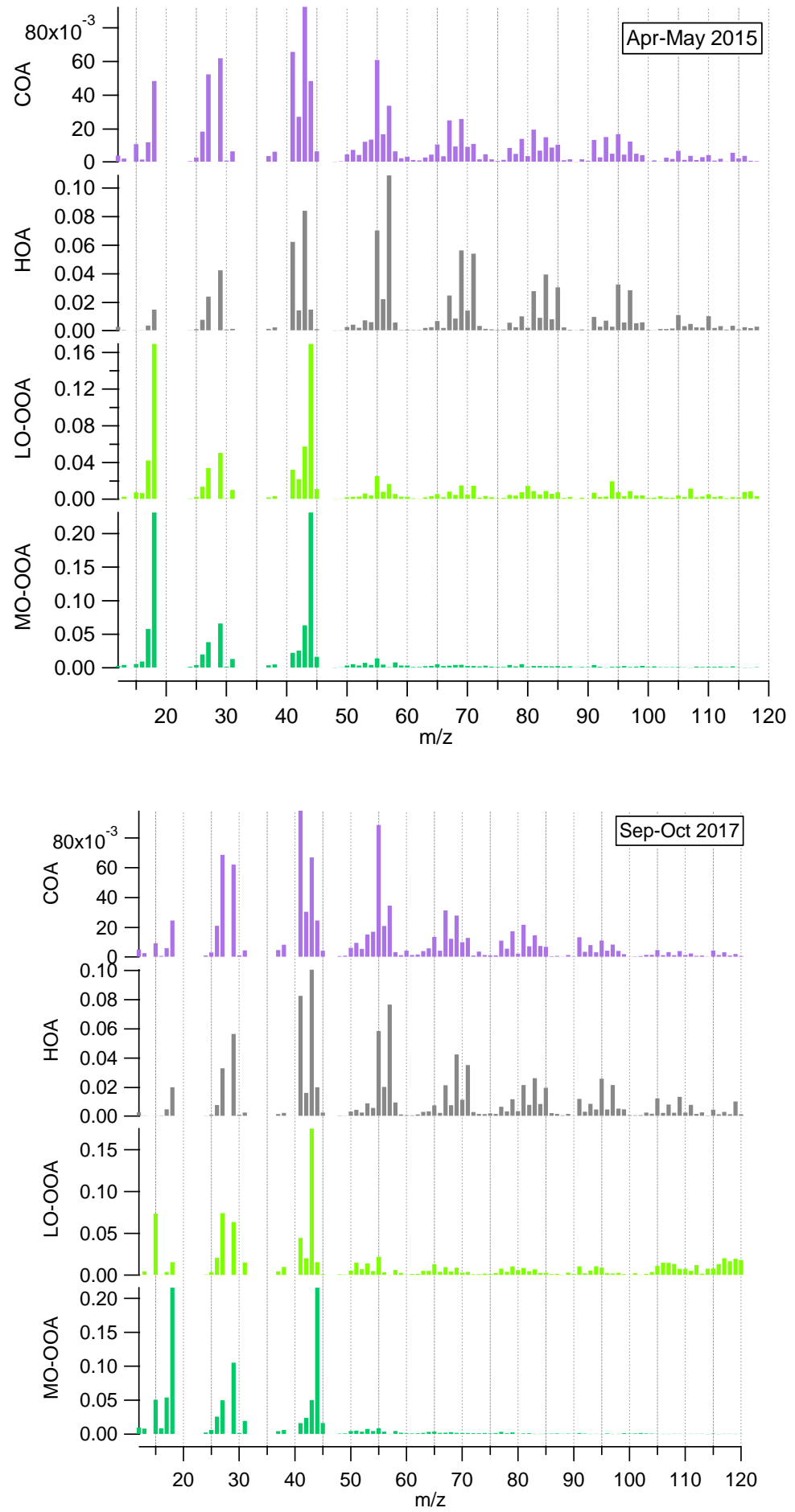

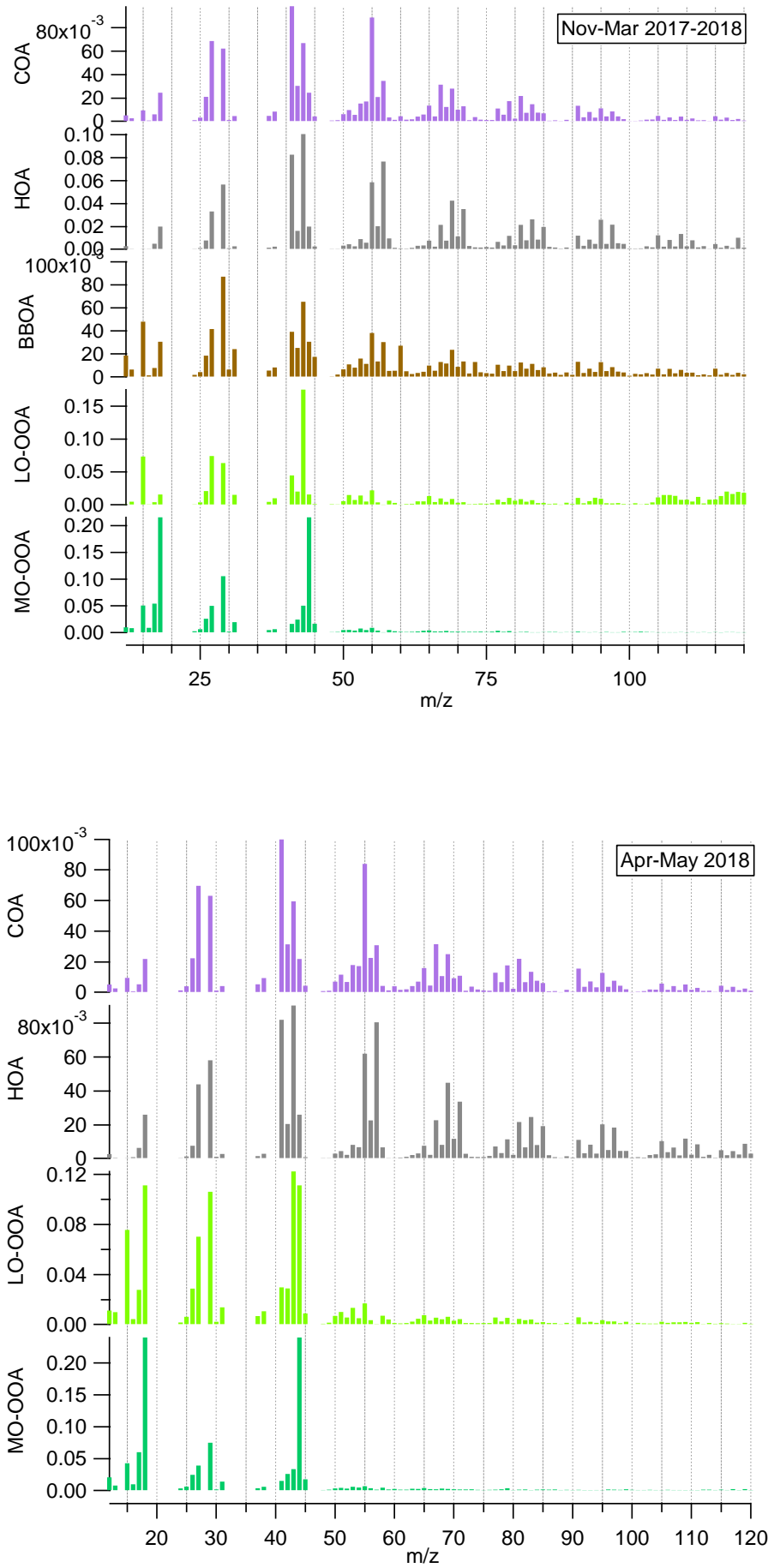

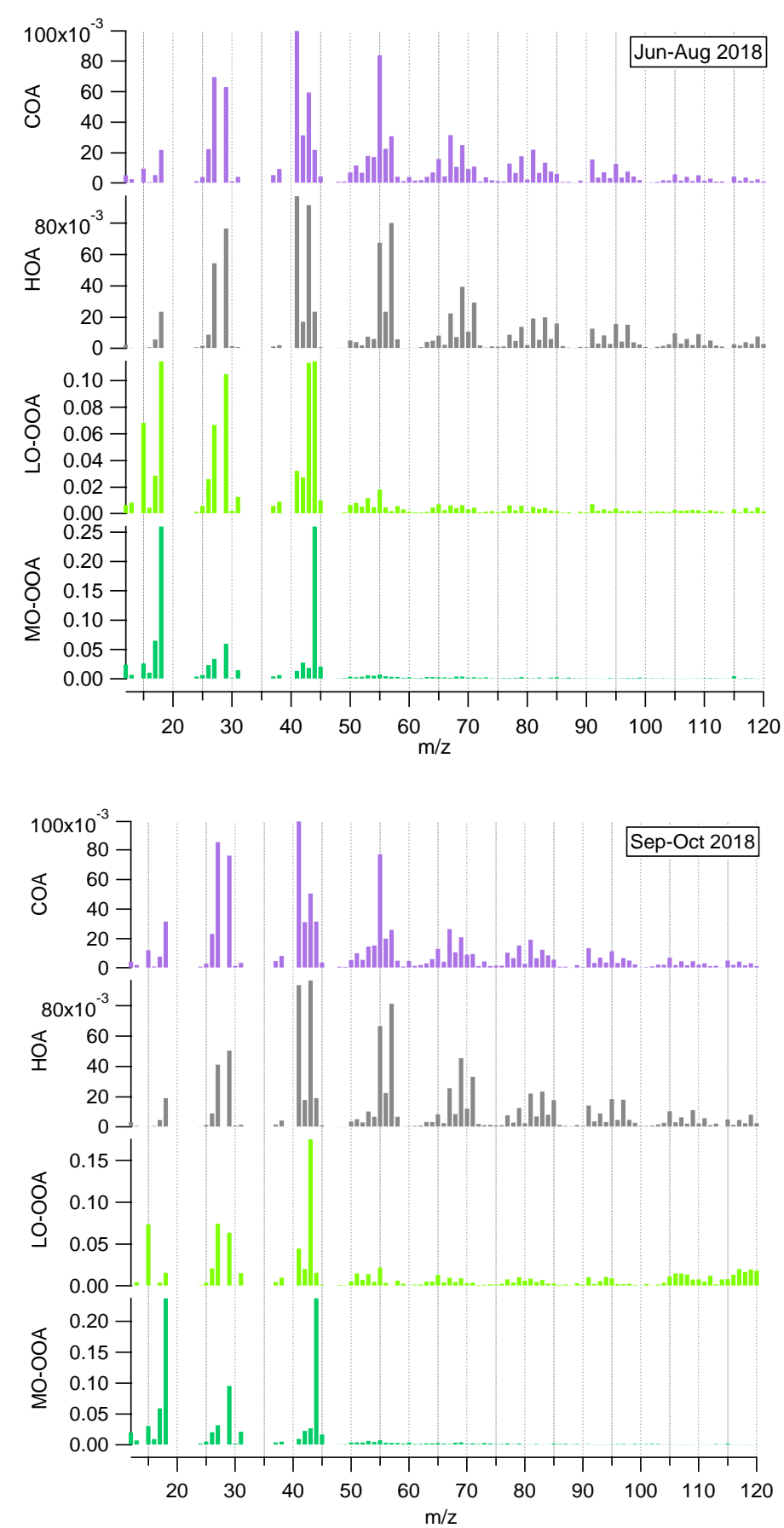

Figure S 10. Seasonal profiles of subperiods of periods A and B by chronological order. 


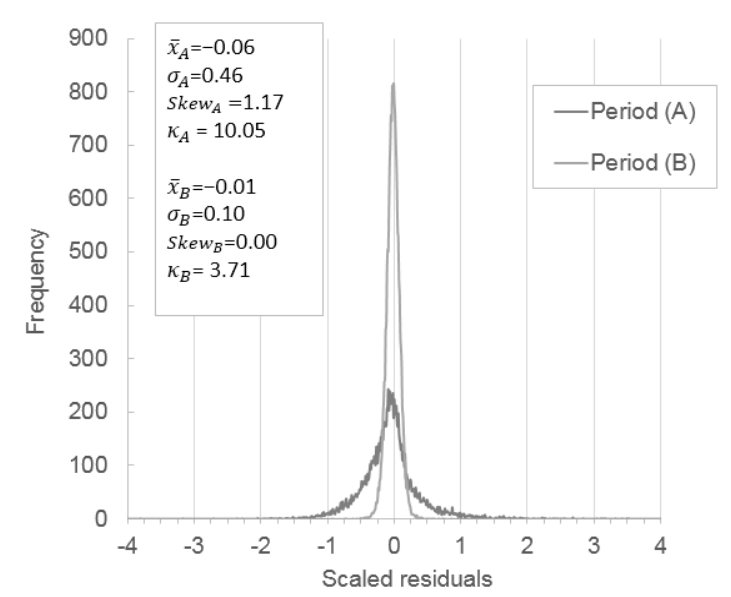

Figure S 11. Residual scaled distributions of PMF OA source distribution using ME-2 approach for periods A and $B$. Parameters of both distributions (mean, standard deviation and skewness and kurtosis coeffcients) are listed.

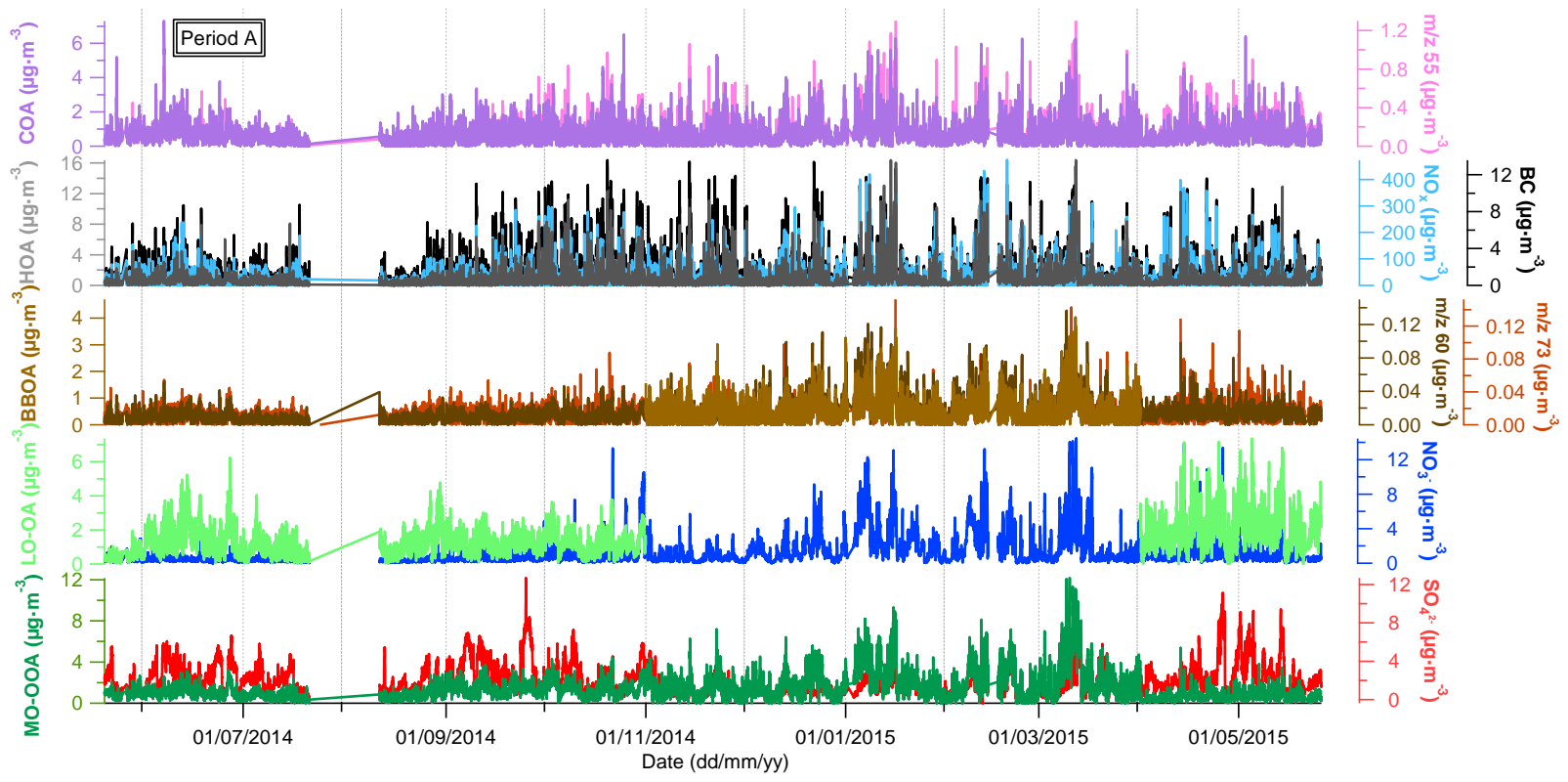




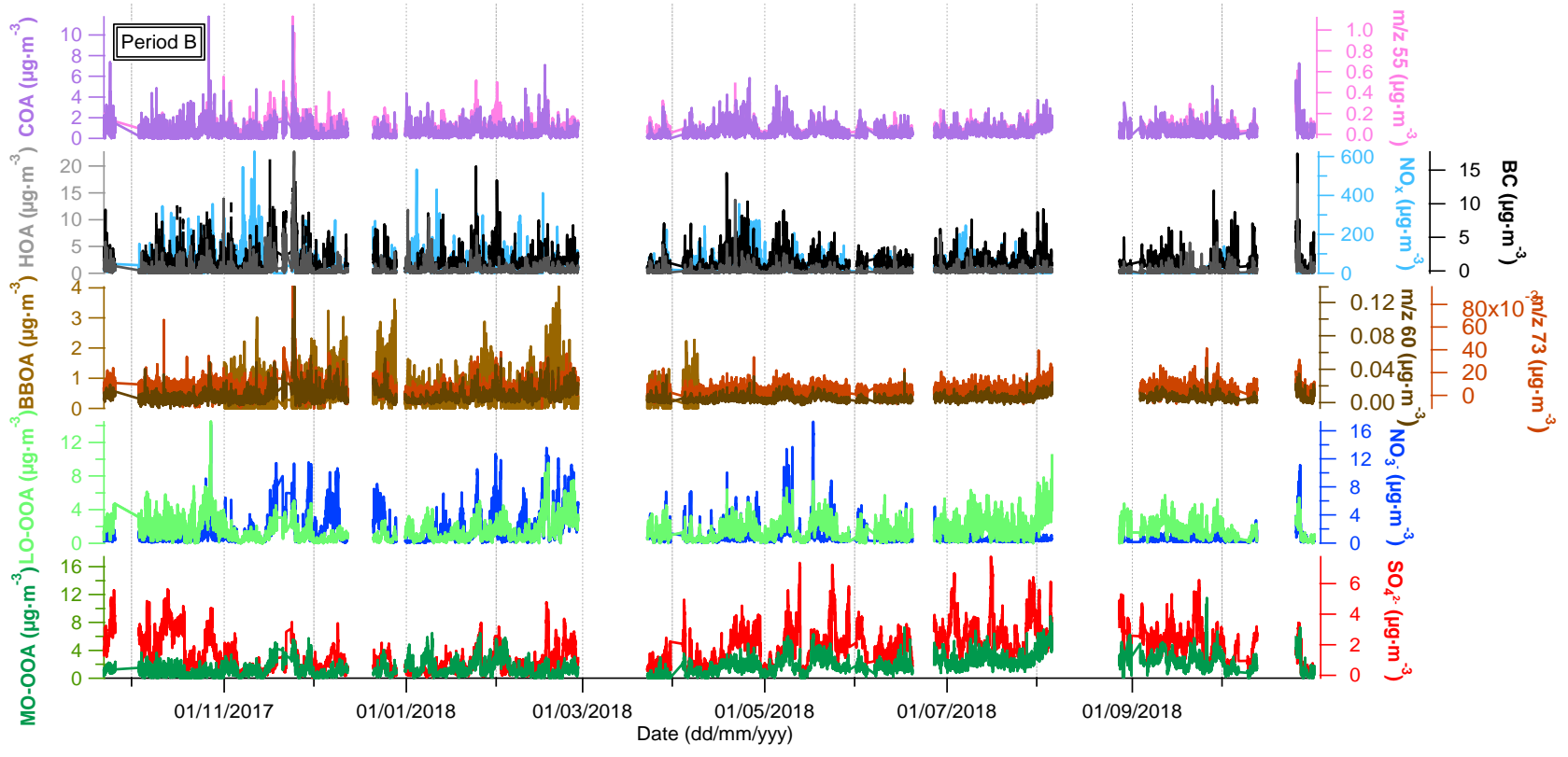

Figure S12. Time series of apportioned OA sources (left axis) and their markers (right axis) in period A and B.

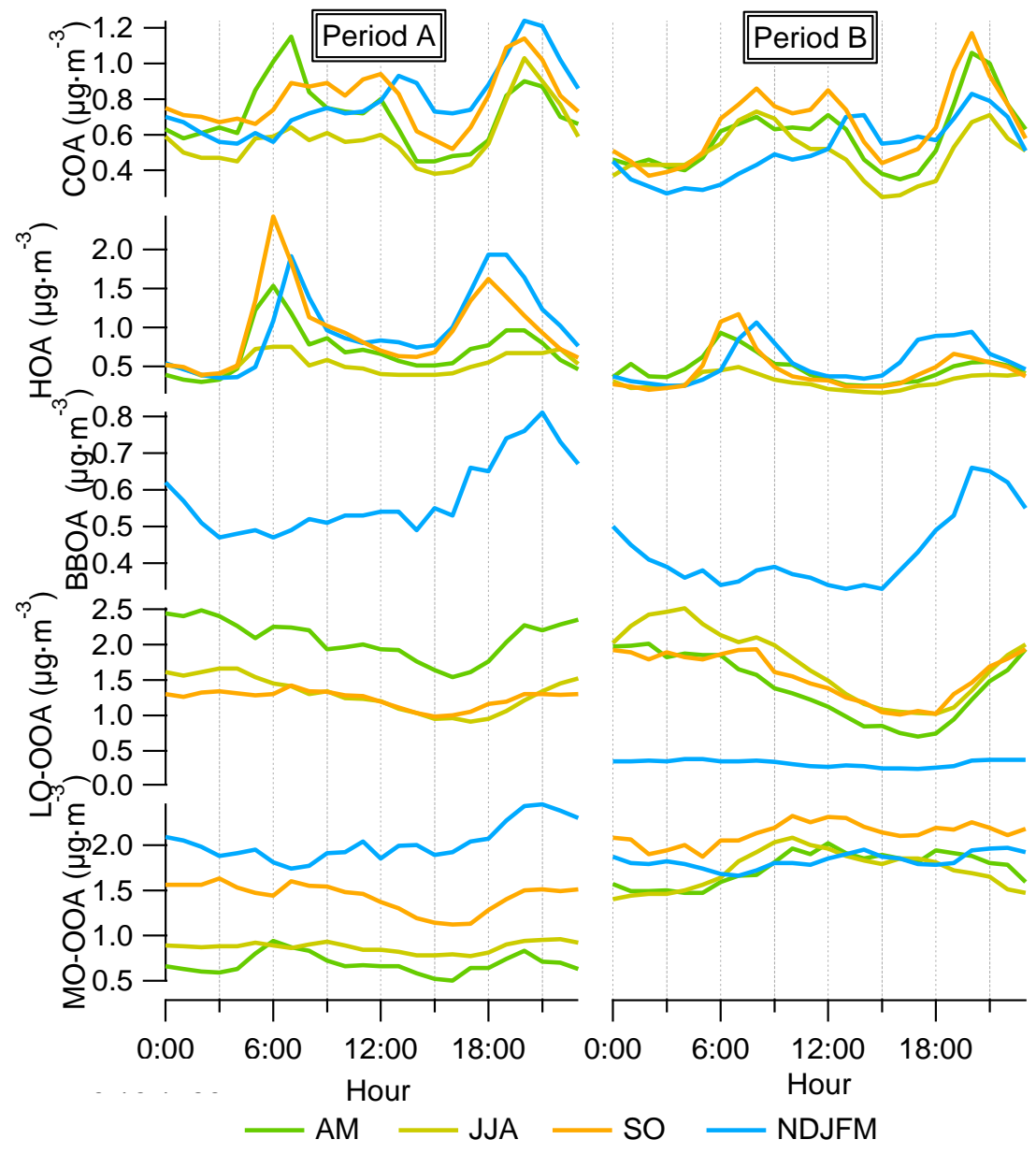

Figure S13. Diel cycle sorted by seasons for the five OA factors for period A and B. Note that the $y$-axis does not start at zero for the sake of clarity. 


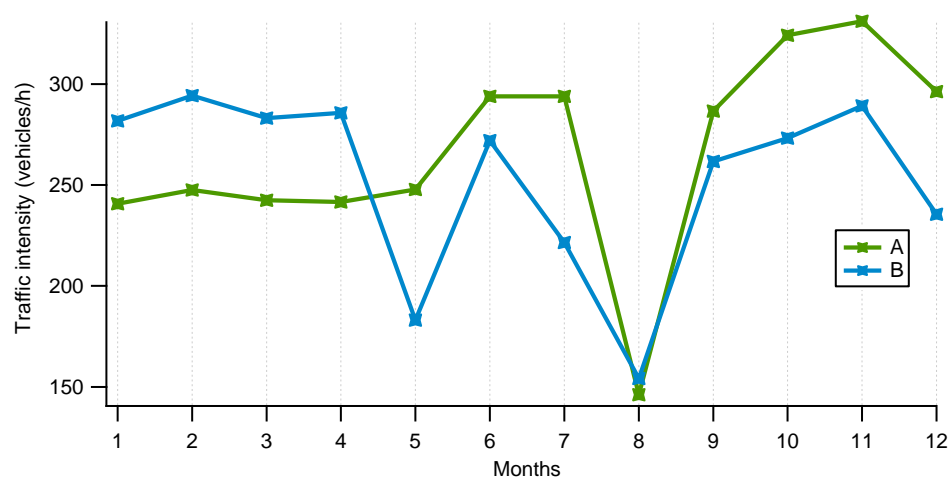

Figure S 14. Mean monthly pattern of traffic intensity at $400 \mathrm{~m}$ away from site for period $A$ and $B$.

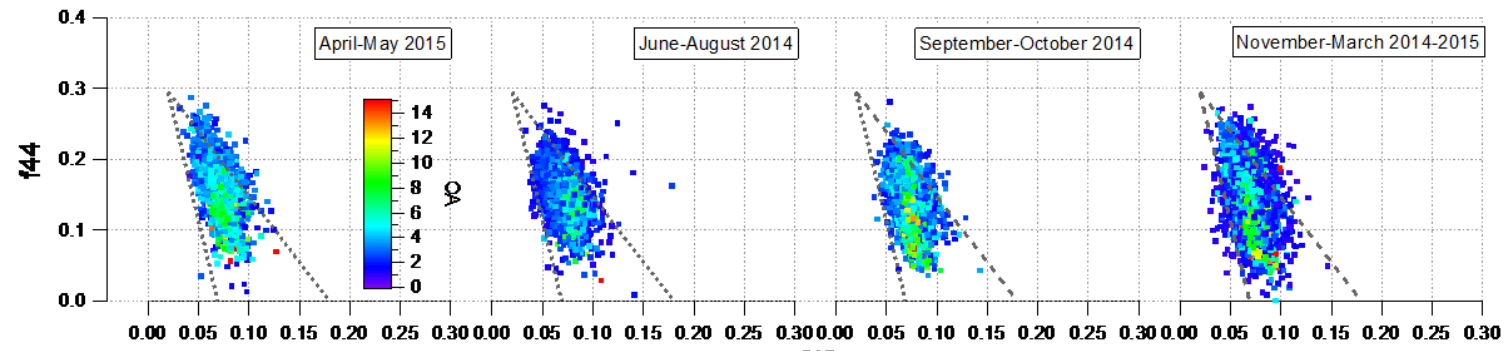

$\mathbf{f 4 3}$

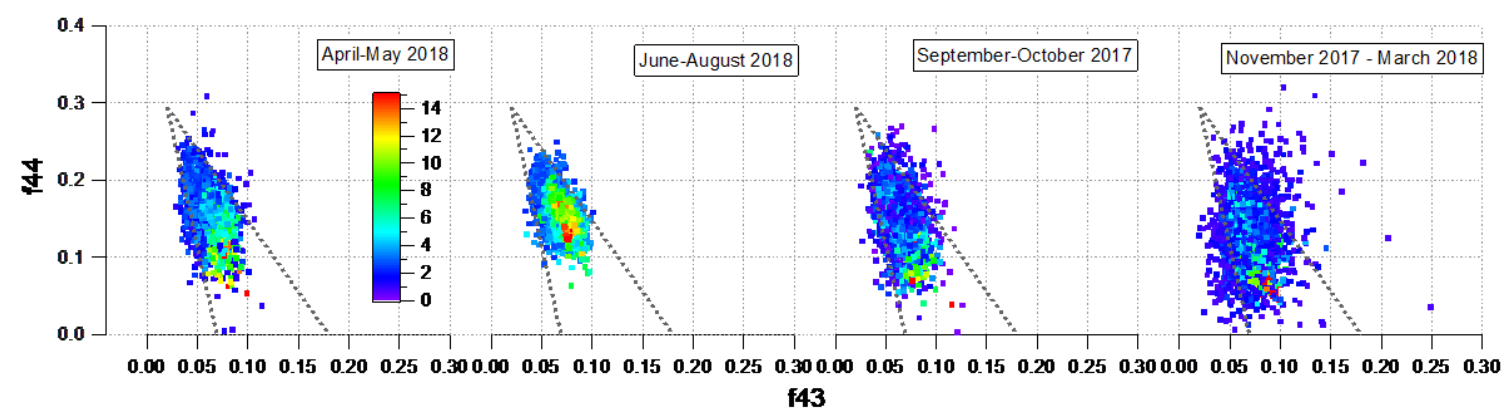

Figure $S$ 15. Seasonal scatterplot of $f 44$ and $f 43$ arranged colored by $O A$ concentrations. Top and bottom graphs correspond to period A and B respectively. Dashed lines correspond to the triangle plot (Ng et al., 2010).

(a) 

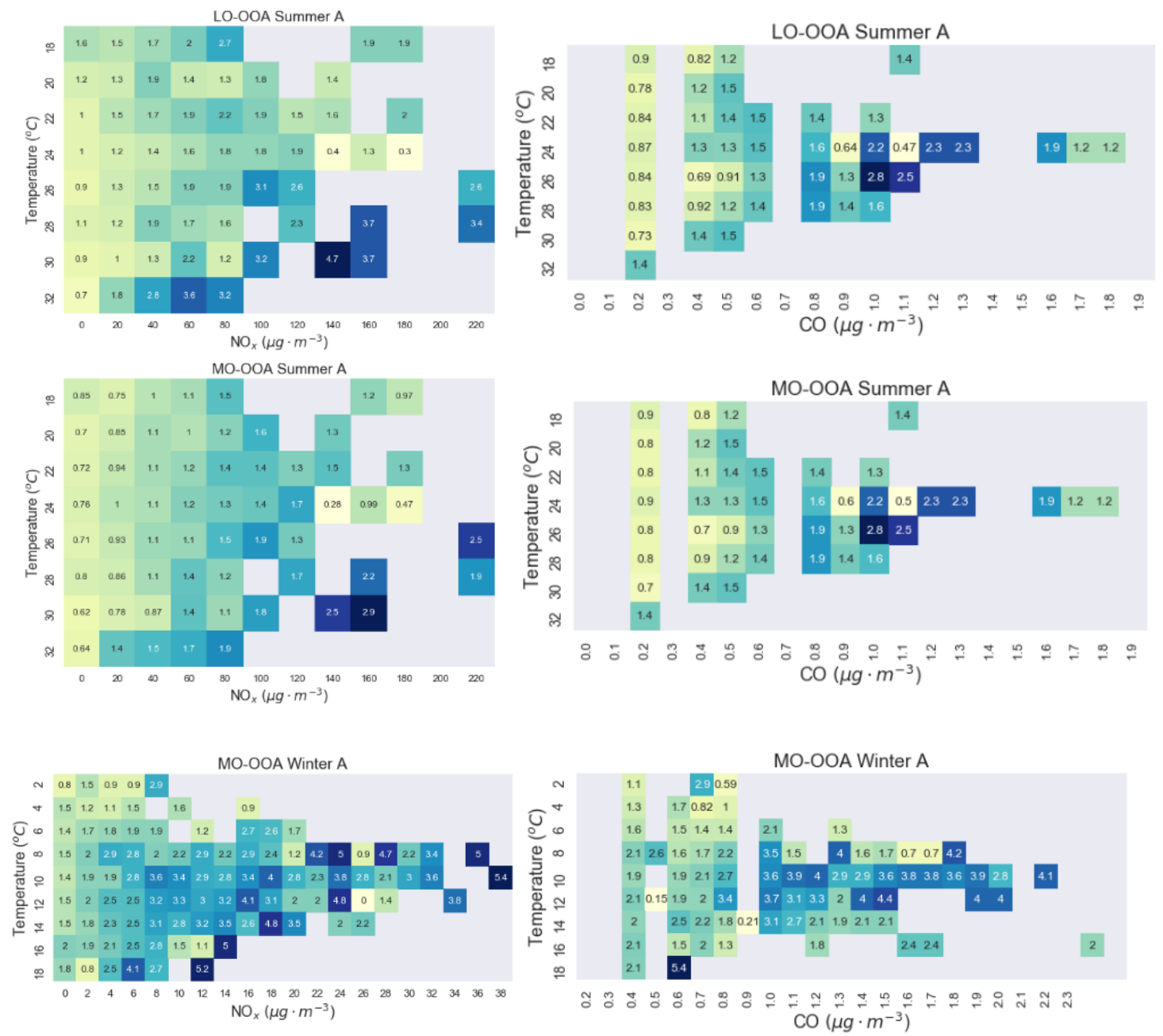

(b) 

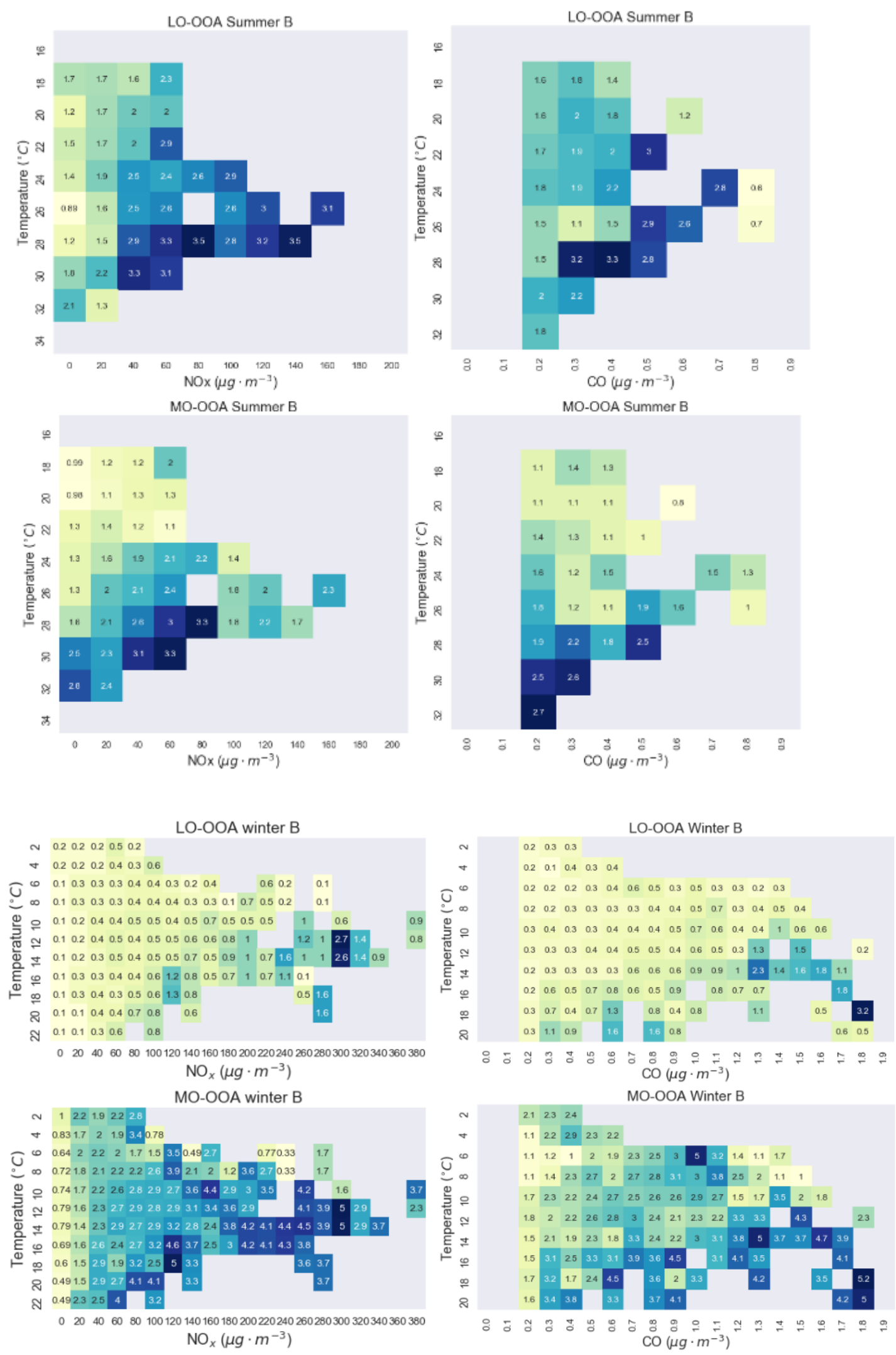

Figure S 16. Heatmap of Temperature vs. NOx for summer (JJA) and winter (DJF) and LO-OOA and MO-OOA respectively for period A (a) and B (b). Figure $\mathrm{S} 1$. Ozone $\left(\mathrm{O}_{3}\right)$ diel cycle for seasons in period A (top) and period B (bottom) at Palau Reial (PR) and Montseny (MSY). 

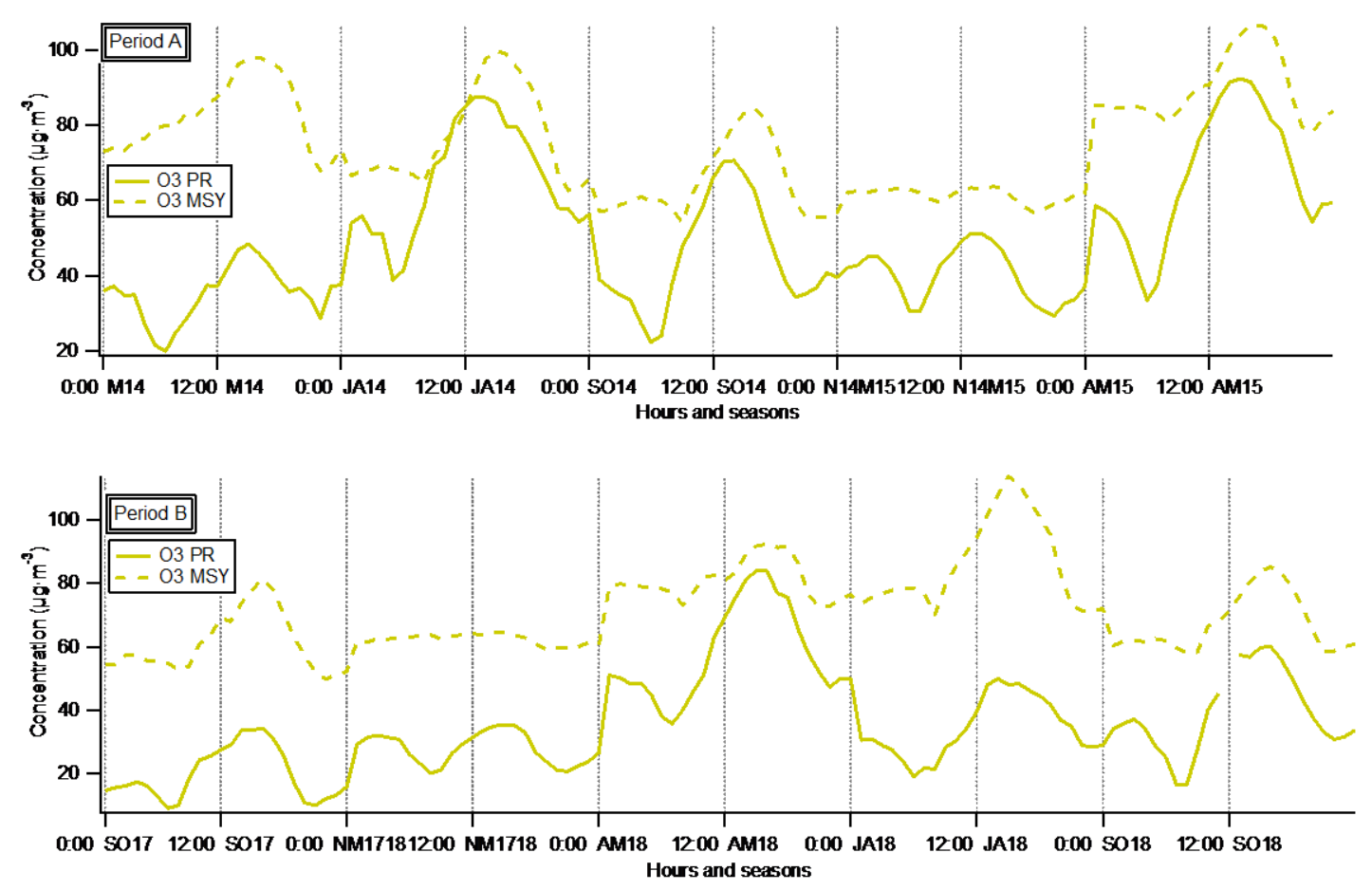

Figure S 17. Ozone diel cycle for seasons in period A and period B at Palau Reial (PR) and Montseny (MSY).

(a) (b)
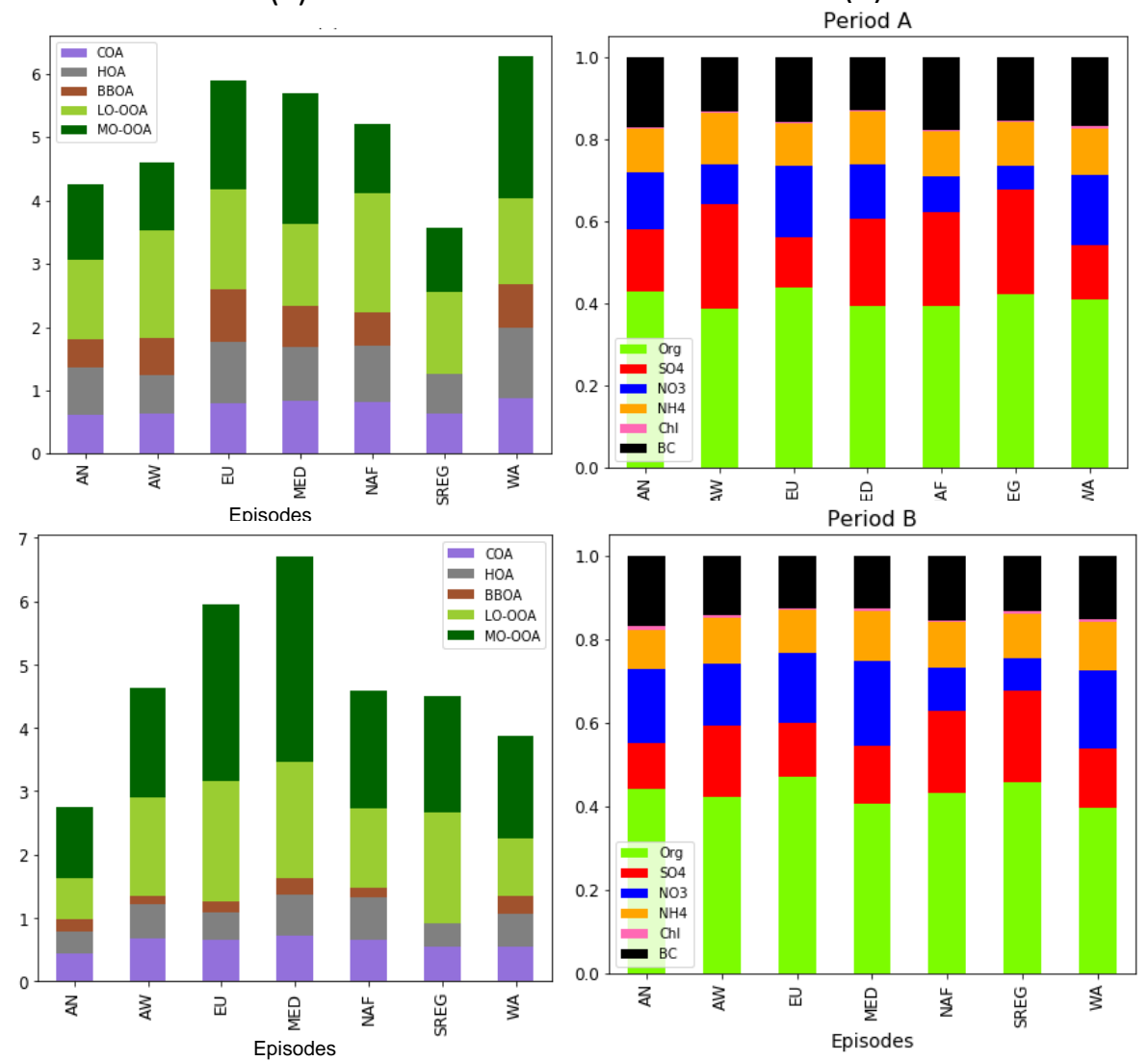

Figure S18. (a) Mean concentrations per each factor grouped by episodes. (c) Relative concentrations of NR-PM1 components grouped by episodes from left to right and for period A (top) and B (bottom). 


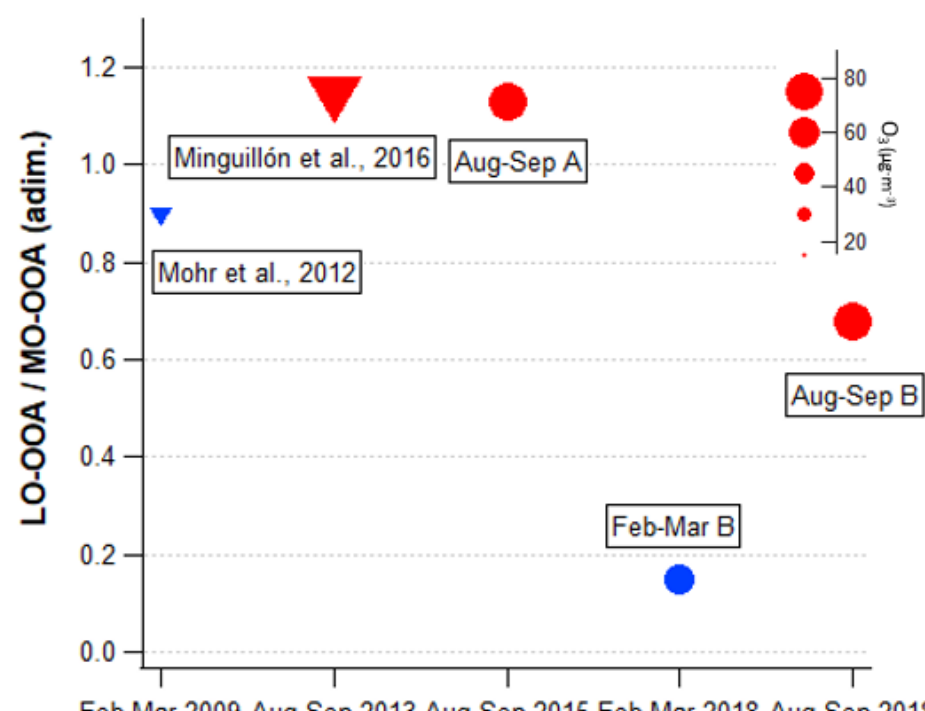

Time

Figure S19. Scatterplot of the LO-OOA-to-MO-OOA ratio vs. time as a function of $\mathrm{O3}$ concentrations (marker size) for the present and previous studies in February-March (blue markers) and August-September (red markers). Note that the Feb-Mar from period A was not included as only one OOA was retrieved.

\section{Section 2. Off-line measurements methodology}

$\mathrm{PM}_{1}$ samples were collected on $150 \mathrm{~mm}$-diameter quartz fiber filters using sequential automatic high-volume samplers Digitel (DHA 80, $30 \mathrm{~m}^{3} \cdot \mathrm{h}^{-1}$ ). The sampling time was 24 hours midnight to midnight and concentrations were assigned to the start date. Sampling frequency was 1 every 3 days. PM1 levels were determined gravimetrically by conditioning and weighting the filters before and after sampling. Due to problems with the gravimetric determination, $\mathrm{PM}_{1}$ mass concentration was reconstructed $\left(\mathrm{PM}_{1}\right.$ reconstructed) by the addition of all components and an estimation of $25 \%$ to account for water.

A complete chemical analysis of the collected $\mathrm{PM}_{1}$ samples was carried out. A quarter of the filter was used for an acidic digestion $\left(\mathrm{HNO}_{3}: \mathrm{HF}: \mathrm{HClO}_{4}\right)$ following the methodology devised by Querol et al., 2001. The resulting solution was analysed by means of Inductively Coupled Plasma Atomic Emission Spectroscopy (ICP-AES, IRIS Advantage TJA solutions, THERMO) and Inductively Coupled Plasma Mass Spectrometry (ICP-MS, X Series II, THERMO) for the major and trace elements concentration determination, respectively. Few mg of the reference material NIST $1633 \mathrm{~b}$ were added to a fraction of laboratory blank filters to check the accuracy of the analysis of the acidic digestions.

A quarter of the filter was water extracted and the concentrations of $\mathrm{NO}_{3}{ }^{-}, \mathrm{SO}_{4}{ }^{2-}$, and $\mathrm{Cl}^{-}$were determined by Ion Chromatography (HPLC) using a WATERS ICpakTN anion column with a WATERS 432 conductivity detector. The concentration of $\mathrm{NH}_{4}{ }^{+}$was determined with a Selective Electrode (MODEL 710 $\mathrm{A}+$, THERMO Orion). $\mathrm{SO}_{4}{ }^{2-}$ concentrations in the present study were those calculated from $\mathrm{S}$ concentrations determined by ICP-AES, in agreement with all previous works by the research group.

A rectangular portion $\left(1.5 \mathrm{~cm}^{-2}\right)$ of the remaining filter was used for the analysis of organic carbon (OC) and

175 elemental carbon (EC) by thermal-optical methods using a SUNSET OCEC analyser following the EUSAAR 2 protocol (Cavalli et al., 2010).

One blank filter was kept for each set of ten filters. Blank concentrations were subtracted from the total concentration measured for each sample, thus giving ambient concentrations. 